\title{
Pulsed positive discharges in air at moderate pressures near a dielectric rod
}

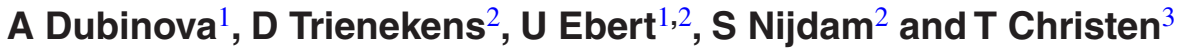 \\ ${ }^{1}$ Centrum Wiskunde \& Informatica, Amsterdam, The Netherlands \\ 2 Technische Universiteit Eindhoven, Eindhoven, The Netherlands \\ 3 ABB Corporate Research, Baden-Daettwil, Switzerland \\ E-mail: a.dubinova@cwi.nl
}

Received 12 February 2016, revised 1 July 2016

Accepted for publication 15 August 2016

Published 9 September 2016

\begin{abstract}
We study pulsed positive discharges in air in a cylindrically symmetric setup with an electrode needle close (about $1 \mathrm{~mm}$ ) above the top of a dielectric cylindrical rod of $4 \mathrm{~mm}$ in diameter mounted at its bottom on a grounded plate electrode. We present ICCD (intensified chargecoupled device) pictures and evaluations of experiments as well as simulations with a fluid discharge model; the simulations use cylindrical symmetry. In the experiments, there is an initial inception cloud phase, where the cylindrical symmetry is maintained, and later a streamer phase, where it is broken spontaneously. At 75-150 mbar, discharges with cylindrical symmetry are not attracted to the dielectric rod, but move away from it. The dielectric rod plays the sole role of an obstacle that shades (in the context of photoionization) a cone-shaped part of the inception cloud; the cone size is determined by the geometry of the setup. The material properties of the dielectric rod, such as its dielectric permittivity and the efficiency of the photon induced secondary electron emission do not have a noticeable effect. This is due to the abundance of photoionization in air, which supplies a positive discharge with free electrons and allows it to propagate along the electric field lines. Using some simple field calculations, we show that field enhancement due to dielectric polarization does not play a significant role in our geometry as long as the discharge maintains its cylindrical symmetry. The field component towards the rod is insufficiently enhanced to cause the discharge to move towards the rod. Any additional electrons produced by the dielectric surface do not influence this discharge morphology.

This interpretation is supported by both experiments and simulations. At higher pressures (400-600 mbar) or for larger gaps between the needle and the dielectric rod, the inception cloud reaches its maximal radius within the gap between needle and rod and destabilizes there. In those cases, streamer channels are more likely to turn into a surface streamer. All our experiments and simulations were performed at moderate pressures (75-600 mbar), but we expect that the results will be the same for other pressures assuming that all the lengths scales (including the rod) in the setup are rescaled according to the Townsend scaling of the discharge.
\end{abstract}

Keywords: surface discharge, stroboscopic imaging, simulations of streamer discharge

(Some figures may appear in colour only in the online journal)

\section{Introduction}

\subsection{The problem}

The dynamics of a corona discharge near a dielectric is still poorly understood and lacks quantitative prediction despite multiple experimental and theoretical studies [1-4]. Empirically, it is well known that discharges developing near dielectric materials are often precursors to surface discharges that damage the insulation and eventually lead to dielectric breakdown. This detrimental effect is one of the limiting factors in high voltage technology $[5,6]$, which 


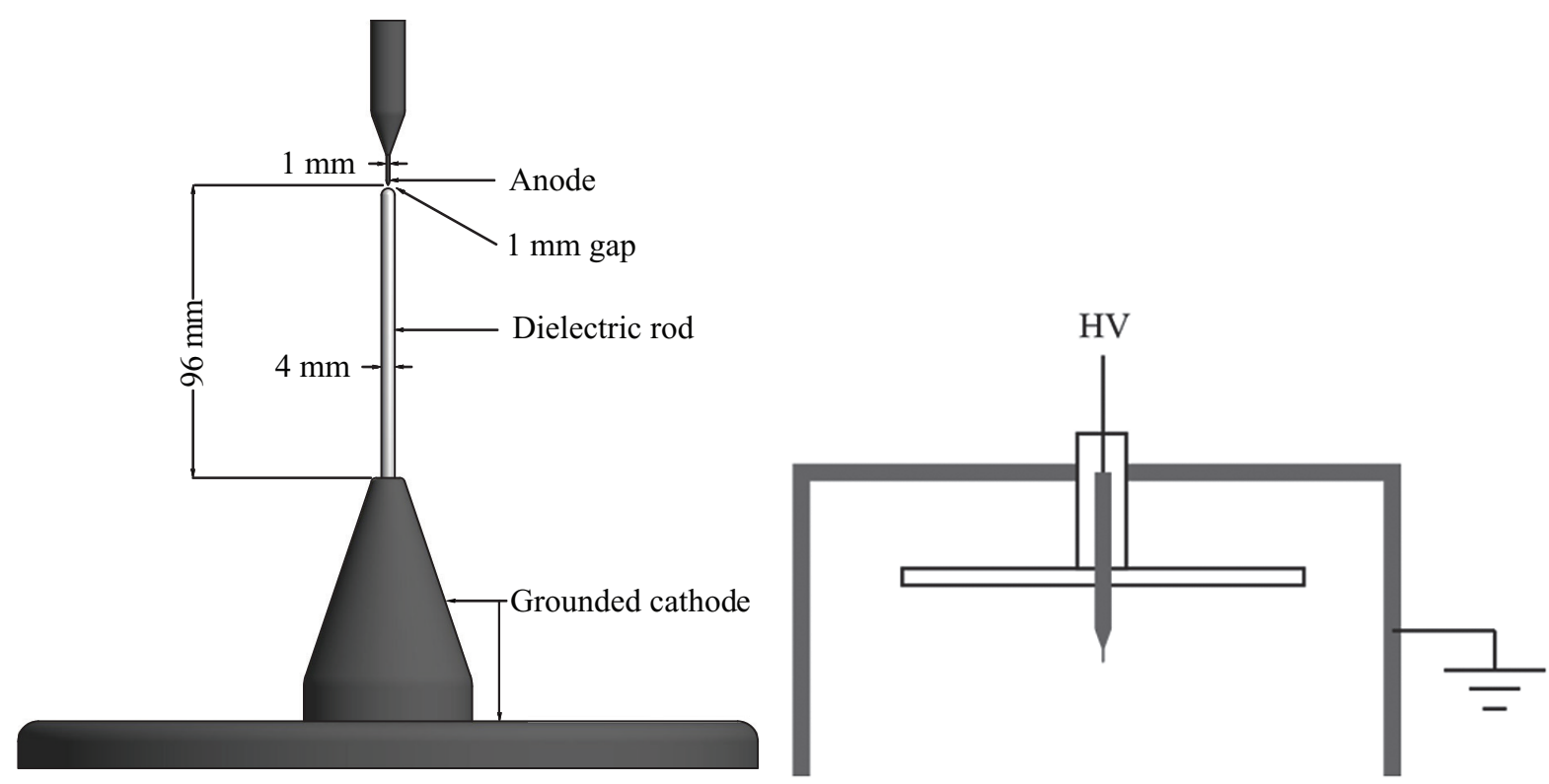

Figure 1. Left: schematic drawing of the experimental setup placed inside a grounded vessel. The dielectric rod is placed directly under the needle. Right: the top half of the vessel, with the high voltage vacuum feedthrough used in the experiments. The white parts indicate insulating material.

necessitates improved understanding of discharges near dielectrics.

In several experiments, streamer discharges were observed to have an affinity to propagate along dielectric surfaces rather than through the background gas only [1, 7-10]. This affinity for a dielectric surface was reported to depend on gas composition [8,11-13], pressure [14], discharge gap geometry [2] and the properties of the dielectric [3, 9, 10]. It is a challenge to understand the physics of discharge interaction with dielectrics due to the interplay and competition of a large number of mechanisms and parameters. Another challenge stems from the intrinsically three-dimensional dynamics of streamer discharges near a dielectric surface, which can rarely be described with a two-dimensional model.

Setups similar to ours have been studied before, for example in $[9,10]$, where two types of streamers in the discharge are identified. One propagates along the surface of a dielectric rod-the surface streamer, and the other streamer propagates in the surrounding bulk air. These two streamers of the discharge exhibit different properties. The streamer in the bulk air propagates slightly slower than it does in the absence of the rod. Besides, its velocity in the presence of the rod is almost independent of the dielectric properties of the rod. The surface streamer propagates significantly faster than the streamer in air, and its velocity depends on the dielectric permittivity of the rod and the surface properties. In general, higher velocities are reported for surface discharges also in other setups $[2,7,15,16]$.

\subsection{Our cylindrically symmetric set-up}

In the present study, we designed a cylindrically symmetric setup that can be realized both in experiments and in simulations [17]. In this manner, the cylindrically symmetric simulation can be compared quantitatively with the experiment, as

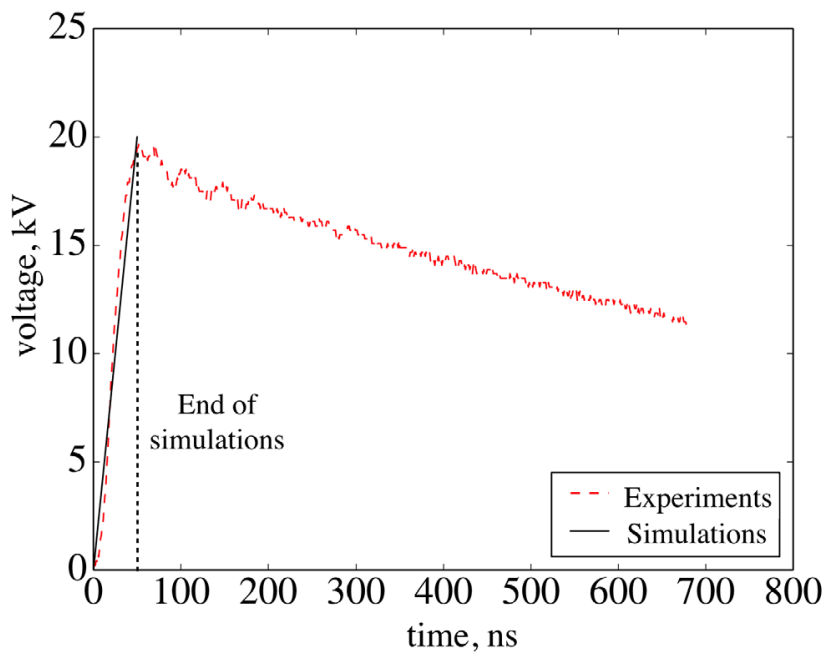

Figure 2. Voltage pulse. The red curve indicates the voltage pulse used in experiments. The black line shows the voltage pulse used in simulations. Discharges are simulated only on the rising slope of the voltage pulse.

long as the actual experimental discharge does not break the cylindrical symmetry.

The geometry of our setup is shown in figure 1. A cylindrically symmetric electrode needle with a sharp tip is placed at a short distance right above a long cylindrical dielectric rod. The needle is inserted into a high voltage vacuum feedthrough, that isolates it from the grounded vessel. The rod touches or is embedded into a grounded electrode (for simulations or experiments, respectively). Experiment or simulations are performed in a large cylindrically symmetric conducting vessel that is at ground potential as well.

A positive voltage pulse (see figure 2) is applied to the needle electrode, and a positive discharge emerges from the 


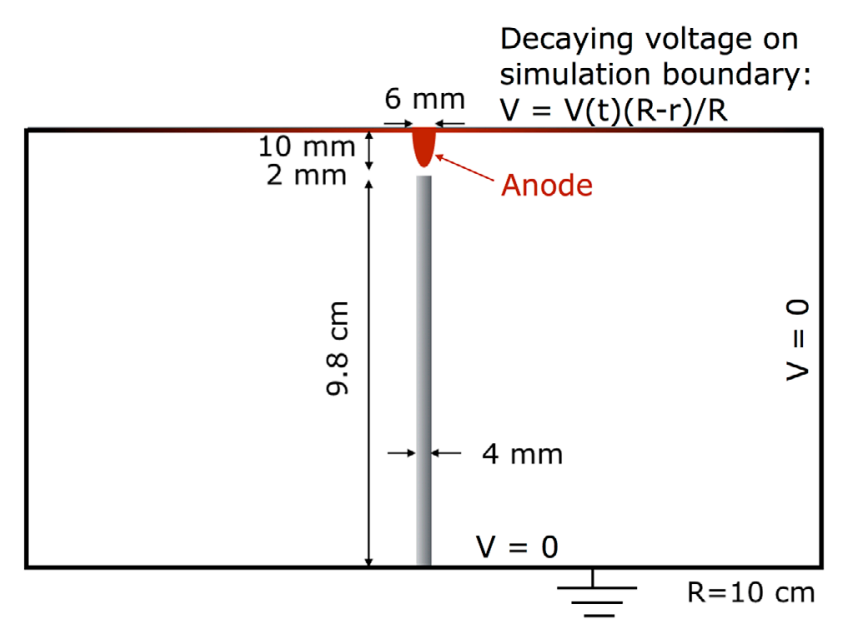

Figure 3. The setup of cylindrically symmetric simulation domain for the needle-to-plate setup with a dielectric rod placed directly under the needle. The color gradient on the anode illustrates that the voltage linearly drops from an applied voltage on the needle to zero on the walls.

needle tip. Such a discharge in air without the dielectric rod evolves in two stages. First a cylindrically symmetric inception cloud is formed that, due to a space charge layer at its expanding surface, can grow up to a maximal radius

$$
R_{\max }=U / E_{c}
$$

where $U$ is the applied potential and $E_{c}$ is the breakdown field, which roughly scales with inverse pressure. This cylindrically symmetric inception cloud then can destabilize and break up into separate streamer filaments that then obviously break the cylindrical symmetry. The concept of inception cloud and its breaking into streamers is described in [18-20].

In our study, we added a dielectric rod at the axis closely below the electrode needle. We expected that the dielectric surface would attract the discharge and maintain the cylindrical symmetry. In order to achieve this goal, we made use of the Townsend scaling of the discharge size with pressure [21]. We thus chose the air pressure so low that the size of the discharge was larger than the diameter of the rod $(4 \mathrm{~mm}$ in our setup) $[22,23]$. That means that the pressure had be lower than about 150 mbar. (However, we expect [21] that our theory would equally apply at 1 bar for a dielectric rod of $0.6 \mathrm{~mm}$ in diameter up to corrections from photoionization quenching. Unfortunately, such a thin rod was not available.)

When the streamer diameter was much smaller than the rod diameter (at higher air pressure), we expected beforehand, that the symmetry would be broken and the streamer would propagate only at one side of the rod, and this happened indeed. However, when the streamer diameter was much larger than the rod diameter (at lower air pressure), it did not propagate over the rod either, neither in a symmetric nor in an asymmetric way.

In this paper, we report and evaluate these experiments and the simulations with cylindrical symmetry, and offer our interpretation.
1.3. Positive discharges and the role of photo-ionization and photo-emission

In our work, we study positive discharges exclusively, because they are more easily producible and controllable in experiments and they are more critical for applications. However, they are more sensitive to sources of free electrons. Positive streamers need free electrons some distance ahead in order to propagate. Among the sources of free electrons are photoionization in air and photon induced secondary electron emission from a dielectric surface (henceforth referred to as photoemission). These sources can be considered nonlocal on the timescales of interest.

Photoemission induced by low energy photons has long been considered a candidate that facilitates streamer propagation along dielectric surfaces and (ultimately) causes a breakdown [8, 24]. The nonlocal nature of photoemission plays an important role here. The required photon energy for this process is low compared to the energy needed for photoionization: typically $<10 \mathrm{eV}[8,25-27]$ compared to $12 \mathrm{eV}$ for photoionization. As a result, the photons are absorbed less in air and can travel larger distances, hitting and liberating electrons from a dielectric surface far away from the head of streamer discharge. A large part of our work is dedicated to studying the influence of photoemission. Other types of secondary electron emission by ion, electron or metastable atom bombardment are local phenomena within the discharge and thus of secondary importance.

Photoemission from the dielectric surface is a competitor to photoionization in air, where photons produced by excited nitrogen molecules hit oxygen molecules and liberate electrons. In pure gases like nitrogen, photoionization is suppressed, and photoemission can be the dominant source of free electrons. In the absence of photoionization, discharges are more localized in space and almost always filamentary, and our 2D cylindrical approximation is no longer justified. Therefore, in this paper we restrict ourselves to discussing discharges in air only.

Even when the 2D cylindrical approximation is valid, the setup is still complex due to the interaction of the parameters at play. As mentioned above, the pressure defines the size of the discharge both in the inception cloud phase and in the streamer phase. The photoionization length also scales inversely proportional with pressure. At the same time, the parameters of our geometry do not scale with pressure. They include the diameter of the rod and the gap between the dielectric rod and the electrode. Besides, there are properties of the dielectric rod that matter. They are the dielectric permittivity and the photoemission efficiency. All the named parameters together influence the dynamics of discharge. And each of the parameters controls a few mechanisms that compete or reinforce each other. Therefore, the task of predicting the outcome of an experiment is challenging.

\subsection{Order of the paper}

In section 2 we discuss the key concepts underlying the physics of a discharge near a dielectric surface and describe 
the model that we developed in order to simulate discharges in the same geometry as in the experiments. Then, in section 3, we describe the experimental setup in detail as well as the experimental results. Section 4 is dedicated to the comparison of our simulations with the experiments. Section 5 is about the interpretation of the results, in which we try to understand how some of the parameters of the setup affect the behavior of a discharge in the presence of a dielectric rod. Finally, in section 6 , we draw the conclusions.

\section{Modeling and simulations}

\subsection{Physical model}

We study positive streamer discharges in artificial air $(80 \%$ $\mathrm{N}_{2}$ and $20 \% \mathrm{O}_{2}$ ) at $75-150 \mathrm{mbar}$ and $300 \mathrm{~K}$ by reproducing the experimental setup in cylindrical needle-to-plate geometry (see figure 3). A needle electrode of fixed potential and a dielectric rod are included into the simulation domain. The dielectric permittivity of the rod was set to 4 and 8 in accordance with the experiments in section 3 . The boundaries of the domain are sufficiently far away from the area of streamer propagation and the electric field on the boundaries does not influence streamer behavior.

In the simulations we consider a single voltage pulse from the repetitive discharges of the experiments. The voltage rises from zero to $20 \mathrm{kV}$ within $50 \mathrm{~ns}$ and then slowly decreases as shown in figure 2 . The repetition frequency of the pulses is 1 Hz. In simulations, we study streamer development only on the rising part of the voltage pulse.

\subsection{Fluid model}

We simulate discharges with the diffusion-drift-reaction model of streamer discharges in local field approximation with cylindrical symmetry and with photoionization included. For a review of fluid models for streamer discharges, see, e.g. [28].

The equations are discretized on a static nonuniform grid. The grid is refined in the area where a discharge is expected to propagate. The size of the finest grid cells is $9 \mu \mathrm{m}$. Away from the area of streamer propagation grid cells quadratically increase in size up to $0.5 \mathrm{~mm}$ on the top and side boundaries, and $5 \mathrm{~mm}$ on the bottom boundary of the domain.

The transport and reactions of electrons are governed by the continuity equation for the electron density $n_{\mathrm{e}}$

$$
\begin{aligned}
\frac{\partial n_{\mathrm{e}}}{\partial t}= & \nabla \cdot\left(n_{\mathrm{e}} \mu_{\mathrm{e}}(E) \mathbf{E}+D_{\mathrm{e}}(E) \nabla n_{\mathrm{e}}\right) \\
& +S_{\mathrm{i}}+S_{\mathrm{ph}}+S_{\mathrm{pe}}
\end{aligned}
$$

where $\mathbf{E}$ is the electric field and $E=|\mathbf{E}|, S_{\mathrm{i}}$ is the effective impact ionization source term (including 2- and 3-body attachment), $S_{\mathrm{ph}}$ is photoionization in air, and $S_{\mathrm{pe}}$ is photon induced secondary electron emission from the dielectric rod; $D_{\mathrm{e}}(E)$ and $\mu_{\mathrm{e}}(E)$ are field dependent electron diffusion coefficient and mobility. All transport and reaction coefficients are calculated with BOLSIG+ solver [29] using Phelps

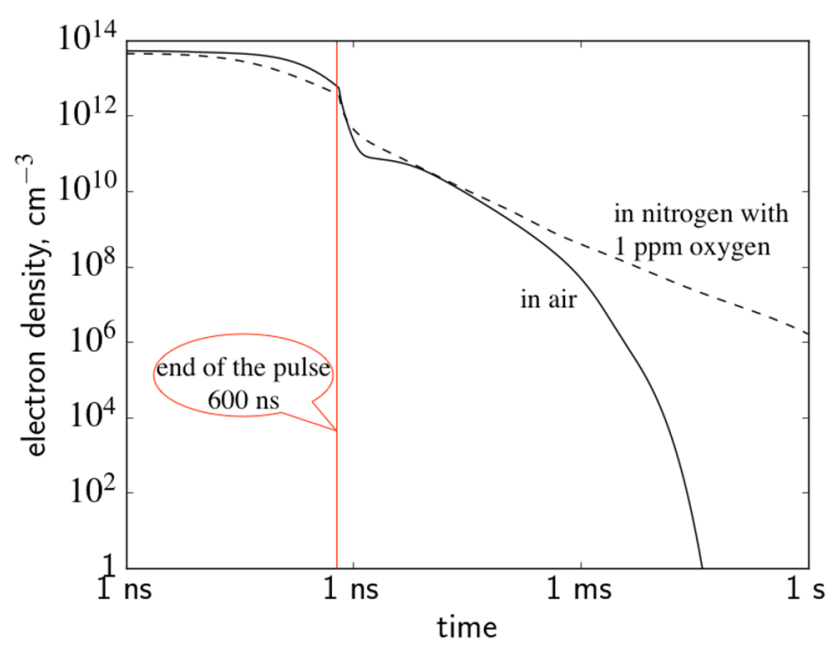

Figure 4. The electron density calculated in air and in nitrogen with $1 \mathrm{ppm}$ oxygen as a function of time at $150 \mathrm{mbar}$ (on the logarithmic scale). Pulse duration $600 \mathrm{~ns}$, initial electron density $9 \times 10^{11} \mathrm{~cm}^{-3}$, maximum electric field on streamer tip: $20 \mathrm{kV} \mathrm{cm}^{-1}$, field in the streamer channel: $0.75 \mathrm{kV} \mathrm{cm}^{-1}$.

database, retrieved on July 31, 2014. To all outer boundaries Neumann boundary conditions are applied. Under the assumption that ions are immobile (they would move $0.1 \mathrm{~mm}$ or 1 grid cell) on the timescale of streamer development (the first $20 \mathrm{~ns}$ ), a similar equation for positive and negative ion densities $N_{\mathrm{i}}^{ \pm}$is given by

$$
\frac{\partial\left(n_{\mathrm{i}}^{+}-n_{\mathrm{i}}^{-}\right)}{\partial t}=S_{\mathrm{i}}+S_{\mathrm{ph}}+S_{\mathrm{pe}} .
$$

We assume for simplicity that electrons, when reaching the dielectric rod, attach to the surface. In general, their attachment probability should be considered [30], but for thermal electrons it is close to 1 . The electrons accumulated on the dielectric surface are treated as charge density sitting in the surface layer which is one cell wide; they do not move, nor react. Conceptually, this approach is different from treating the surface charge as a surface boundary condition, but in practice for a fine enough grid the difference is negligible.

\subsection{Electric field}

The electric field distribution $\mathbf{E}$ is calculated by solving Poisson's equation for the electric potential $\psi$ :

$$
\begin{aligned}
\nabla\left(\epsilon_{0} \epsilon \nabla \psi\right) & =e\left(n_{\mathrm{e}}-n_{\mathrm{i}}^{+}+n_{\mathrm{i}}^{-}\right), \\
\mathbf{E} & =-\nabla \psi,
\end{aligned}
$$

where $\epsilon_{0}$ is the permittivity of free space, $\epsilon$ is the relative dielectric permittivity of the dielectric rod, and $e$ is the elementary charge.

The ghost fluid method was used to accurately capture the boundary conditions on the electrode and dielectric curved interfaces based on $[31,32]$. Conceptually, any shape of an electrode and a dielectric can be rendered with this method. For example, the rod is parameterized as a cylinder with a flat top. The needle electrode in our simulations was parameterized 
as an ellipsoid of revolution with a given radius of curvature and a length. Although, the shape of the needle electrode differs from the one used in experiments, it essentially does not influence the discharge propagation. The only purpose of the needle in our experiments is to launch a discharge in an inhomogeneous field, but once the discharge has started, its plasma screens the electrode shape and creates its own self-consistent electric field enhancement at its surface. Therefore, we chose the ellipsoidal shape for the sake of simplicity.

Dirichlet boundary conditions are used in the radial direction and on the sides of the simulation domain. The bottom and the sides of the simulation domain are grounded. Away from the needle in the radial direction the voltage decreases linearly and reaches zero on the sides of the simulation domain (see figure 3). This inhomogeneous Dirichlet boundary condition with the artificial linear voltage drop at the top of the vessel turns out to be convenient in simulations, and the exact shape of the radial drop has not significant influence on the streamer discharge, because the needle tip is far away from the top of the vessel.

\subsection{Photoionization in the bulk gas}

Positive streamer-like discharges need a source of free electrons ahead of them in order to propagate. In the bulk gas this source can be photoionization $S_{\mathrm{ph}}$ in equations (2) and (3) or background ionization (see figure 4). In our experiments, the effect of the background ionization is negligible (see section 2.7). The photoionization in air is possible due to the photons produced in air by molecular nitrogen. They are VUV photons of the 13-14 eV energy range (98-102.5 nm). Oxygen molecules absorb this radiation and produce free electrons [33].

We use the photoionization model developed for air in [34]. In that model, the photoionization source term $S_{\mathrm{ph}}$ is approximated with a weighted sum of the solutions to elliptic equations, as follows

$$
\begin{gathered}
S_{\mathrm{ph}}=\frac{p_{\mathrm{q}}}{p+p_{\mathrm{q}}}\left(A_{1} S_{\mathrm{ph}, 1}+A_{2} S_{\mathrm{ph}, 2}\right), \\
\left(\nabla^{2}-\lambda_{1,2}^{2}\right) S_{\mathrm{ph}, 1,2}=-\left|S_{\mathrm{i}}\right|,
\end{gathered}
$$

where $S_{\mathrm{i}}$ is impact ionization source term from equation (2), $\lambda_{1,2}$ and $A_{1,2}$ are coefficients chosen to fit the experimental data and taken from [34]. With the print errors corrected, the coefficients read $A_{1}=6 \cdot 10^{-5} \mathrm{Torr}^{-2} \mathrm{~cm}^{-2}, A_{2}=3.55 \cdot 10^{-6}$ Torr $^{-2} \mathrm{~cm}^{-2}, \lambda_{1}=0.059$ Torr $^{-1} \mathrm{~cm}^{-1}$ and $\lambda_{2}=0.01$ Torr $^{-1} \mathrm{~cm}^{-1}$. The factor $p_{\mathrm{q}} /\left(p+p_{\mathrm{q}}\right)$ represents the effect of collisional quenching, where $p$ and $p_{\mathrm{q}}=60$ Torr are pressure and the quenching pressure, respectively. The parameters $\lambda_{1,2}$ (proportional to the oxygen content and pressure) define the characteristic length scale of photoionization or photoionization length. In ambient air the photoionization length is about $1.3 \mathrm{~mm}$, and at pressures $75-150 \mathrm{mbar}$, it ranges from $8.7 \mathrm{~mm}$ to $17.3 \mathrm{~mm}$.

In our model, photons and electrons produced by photoionization can be created only outside the dielectric rod and the electrode. After equation (7) are solved everywhere inside the simulation domain, the solutions are set to zero inside the dielectric and inside the electrode. This means that photoionization in some areas is suppressed by shading.

\subsection{Photoemission from the dielectric rod}

A streamer discharge in air can be a source of visible and ultraviolet radiation with photons of energies $2.27-4.63 \mathrm{eV}$ $(280-440 \mathrm{~nm})$ which are produced by the second positive system (SPS) of molecular nitrogen corresponding to the $\mathrm{N}_{2}^{*}\left(C^{3} \Pi_{\mathrm{u}}\right) \rightarrow \mathrm{N}_{2}\left(B^{3} \Pi_{\mathrm{g}}\right)$ transition. These photons are hardly absorbed in air. Radiation due to the first positive system is dominant for sprite discharges in the thin air of the terrestrial mesosphere $[21,35]$ but quenched at higher pressures.

In order to write the kinetic equation of balance for $\mathrm{N}_{2}^{*}\left(C^{3} \Pi_{\mathrm{u}}\right)$, we need to consider the reactions of impact and photo- ionization $(8 a)$ and $(8 b)$ and the competing reaction associated with the interaction of the excited nitrogen with other molecules, which leads to quenching of $(8 c)$ and $(8 d)$. In nitrogen-oxygen mixtures these reactions read [36]:

$$
\begin{gathered}
e+\mathrm{N}_{2} \stackrel{k_{\mathrm{ex}}}{\longrightarrow} \mathrm{N}_{2}^{*}\left(C^{3} \Pi_{\mathrm{u}}\right)+e, \\
\mathrm{~N}_{2}^{*}\left(C^{3} \Pi_{\mathrm{u}}\right) \stackrel{1 / \tau_{0}}{\longrightarrow} \mathrm{N}_{2}\left(B^{3} \Pi_{\mathrm{g}}\right)+h \nu, \\
\mathrm{N}_{2}^{*}\left(C^{3} \Pi_{\mathrm{u}}\right)+\mathrm{N}_{2} \stackrel{k_{\mathrm{q}}^{\mathrm{N}_{2}}}{\longrightarrow} \text { products, } \\
\mathrm{N}_{2}^{*}\left(C^{3} \Pi_{\mathrm{u}}\right)+\mathrm{O}_{2} \stackrel{k_{\mathrm{q}}^{\mathrm{O}_{2}}}{\longrightarrow} \text { products. }
\end{gathered}
$$

where $e$ represents the electron, and h $\nu$ is energy of a photon emitted during deexcitation. Further, $k_{\mathrm{ex}}$ is the rate of excitation reaction $(8 a), \tau_{0}=42 \mathrm{~ns}$ is the radiative lifetime, and $k_{\mathrm{q}}^{\mathrm{N}_{2}}=0.13 \cdot 10^{-10} \mathrm{~cm}^{3} \mathrm{~s}^{-1}$ and $k_{\mathrm{q}}^{\mathrm{O}_{2}}=3.0 \cdot 10^{-10} \mathrm{~cm}^{3} \mathrm{~s}^{-1}$ are the quenching rate constants on the $\mathrm{N}_{2}$ and $\mathrm{O}_{2}$ molecules, respectively. The dependence of the rate constant of electronic excitation on the reduced electric field $k_{\mathrm{ex}}(E / N)$ was calculated using BOLSIG+ [29], and the lifetime and the quenching rate constants were taken from [37].

The density of $\mathrm{N}_{2}^{*}\left(C^{3} \Pi_{\mathrm{u}}\right)$ in reactions $(8 a)-(8 d)$ can thus be calculated by solving the following kinetic equation [36]

$$
\frac{\mathrm{d}\left[\mathrm{N}_{2}^{*}\right]}{\mathrm{d} t}=k_{\mathrm{ex}} n_{\mathrm{e}}\left[\mathrm{N}_{2}\right]-\frac{\left[\mathrm{N}_{2}^{*}\right]}{\tau} .
$$

Here, $\tau$ is the total lifetime of the radiating state, defined as the inverse rate of three parallel decay processes (i.e. via the sum of the three rates)

$$
\frac{1}{\tau}=\frac{1}{\tau_{0}}+k_{\mathrm{q}}^{\mathrm{N}_{2}}\left[\mathrm{~N}_{2}\right]+k_{\mathrm{q}}^{\mathrm{O}_{2}}\left[\mathrm{O}_{2}\right] .
$$

According to equation $(8 b)$, the production rate of the photons can be estimated as 

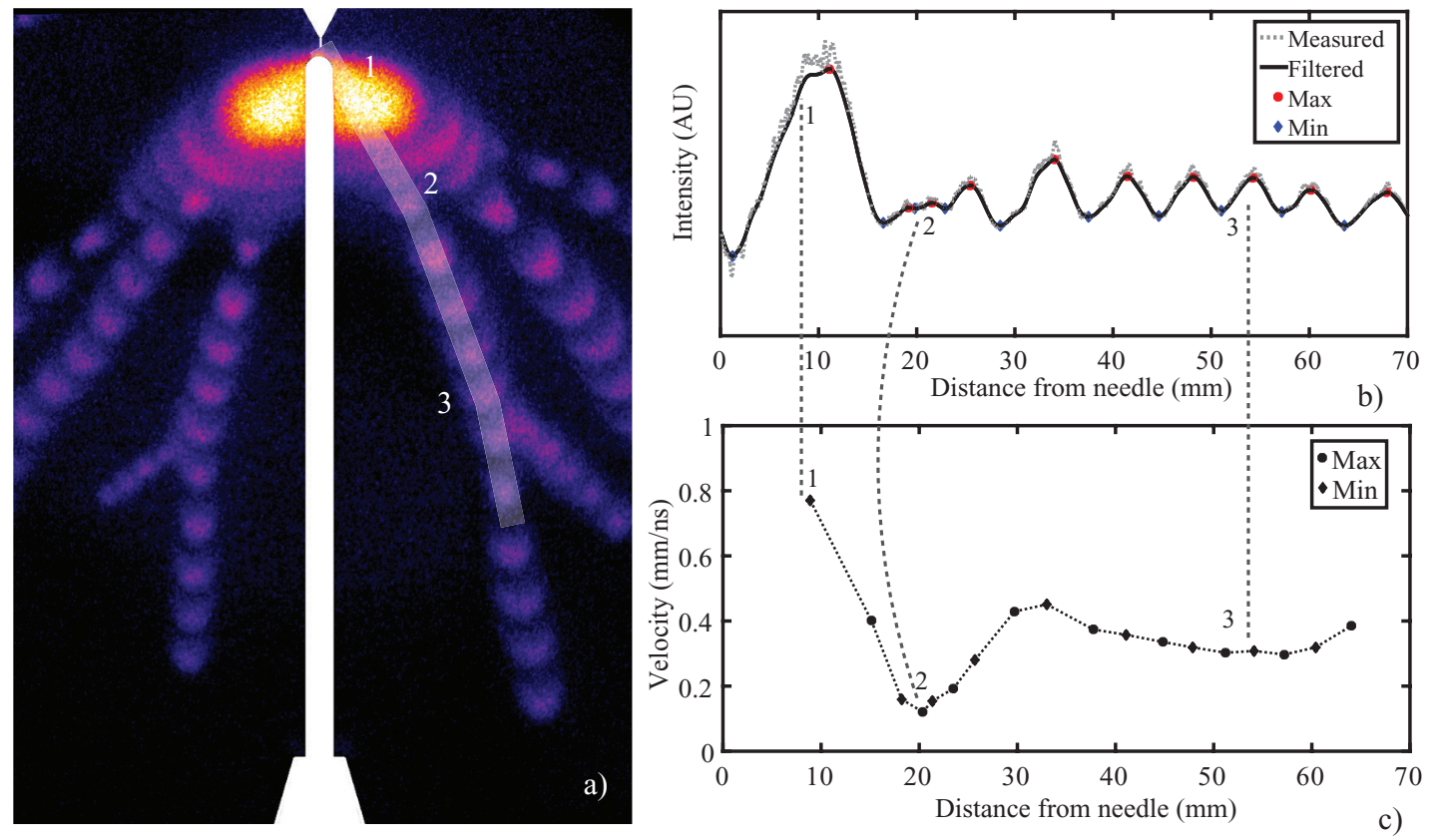

Figure 5. Stroboscopic image of a discharge at 150 mbar with the epoxy resin rod in the discharge gap (a) and the resulting intensity profile (b) and velocity (c). The semi-transparent white region in the stroboscopic image indicates the region where the intensity profile is measured. From this profile, the velocity is calculated. Three distinctive phases can be distinguished: rapid expansion of the inception cloud (1), stagnation of the inception cloud (2), and streamer propagation at steady velocity (3).

$$
P(\mathbf{r}, t)=\frac{\left[\mathrm{N}_{2}^{*}\right]}{\tau_{0}} .
$$

In a quasi-stationary approximation, where $\mathrm{d}\left[\mathrm{N}_{2}^{*}\right] / \mathrm{d} t \equiv 0$, the production of photons can be calculated as follows

$$
P(\mathbf{r}, t)=\frac{\tau}{\tau_{0}} Q(\mathbf{r}, t)
$$

where $Q(\mathbf{r}, t)=k_{\mathrm{ex}} n_{\mathrm{e}}\left[\mathrm{N}_{2}\right]$ is the excitation source term by electron impact. Making use of the fact that $\left[\mathrm{N}_{2}\right]$ and $\left[\mathrm{O}_{2}\right]$ are proportional to total pressure, we can write equation (12) in the same form as in equation (6) for the production of photons in photoionization in air:

$$
P(\mathbf{r}, t)=\frac{p_{\mathrm{q}}}{p+p_{\mathrm{q}}} Q(\mathbf{r}, t),
$$

where $p_{\mathrm{q}}$ essentially stands for collisional quenching. For air at STP for example, $P=0.013 Q$, for air at 100 mbar $P=0.12 Q$, and for $\mathrm{N}_{2}$ at 100 mbar $P=0.42 Q$. Along with photoionization, we assume that the quasi-stationary approximation is valid.

The photons produced in reaction $(8 b)$ are considered the main source of electrons from dielectric surfaces. Since the lower energy photons creating photoemission can travel farther before being absorbed than the higher energy photons creating photoionization (see section 2.4), it is presumed (e.g. in [8]) that an ionization wave next to a dielectric surface will travel with greater velocity than an ionization wave without a surface.

We include photoemission from the dielectric surface $S_{\mathrm{pe}}$ in equation (2). At every time step we calculate the flux of photons onto every point on the dielectric surface. The flux of photoelectrons from the dielectric surface is proportional to the flux of photons with a coefficient $\gamma$ (photoemission yield). Thereby, the emitted electrons are put just outside of the surface and become bulk electrons; ions stay immobile on the surface.

The influence of photoemission on the streamer discharge propagation has previously been studied, for example, in $[38,39]$ where the photon source was integrated directly. We for the first time consider a geometry with a profound shading effect. In the appendix A we describe the implementation of the photoemission. Alternatively, photoemission was studied in the framework of the radiative transfer equation for photon transport [40].

\subsection{The uncertainty about the photoemission yield}

The probability of a photon to liberate an electron is called photoelectron emission yield $\gamma$. It depends on the material of the dielectric, the properties of the dielectric surface (roughness, accumulated charge, etc), and on properties of the plasma produced by a streamer discharge in a gas.

The photoelectron emission is fairly well understood and experimentally measured for metals in vacuum [41]. In gases, and especially when a discharge develops near the emitting surface, the experimental data are scarce. It is argued that the photoemission yield can increase by even an order of magnitude [42] and reach the values from 0.1 to 1.0 for photons with wavelength less than $100 \mathrm{~nm}$. The large values of $\gamma$ are explained by the adsorbed atoms that contaminate the surface. They absorb the resonant radiation produced by a discharge and the resonant state is deactivated in the Auger process. These experiments have been performed in noble gases like $\mathrm{Kr}, \mathrm{Xe}, \mathrm{He}$. 

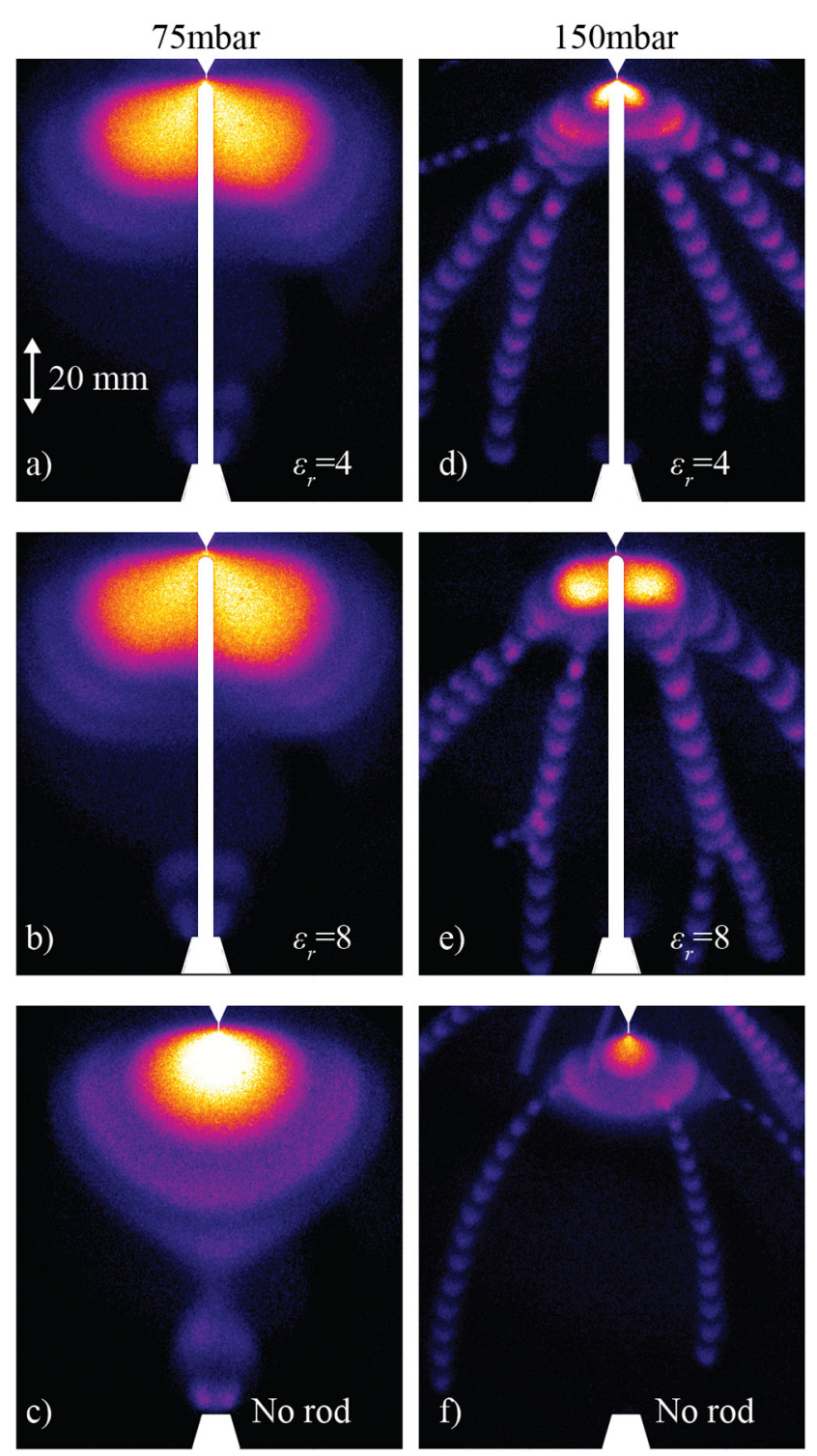

Figure 6. Typical stroboscopic images obtained at 75 mbar (a)-(c) and 150 mbar (d)-(f) with the epoxy resin $\operatorname{rod}(\epsilon=4)$, the $\mathrm{TiO}_{2}$ filled $\operatorname{rod}(\epsilon=8)$, and without rod.

Photoemission from dielectrics in air (or nitrogen-oxygen mixtures) is less understood than photoemission from metals. The development of avalanches in the vicinity of some dielectrics (polyethylene) in pure gases or in vacuum were studied in [4349]. For the photons of $280-440 \mathrm{~nm}\left(\mathrm{SPS} \mathrm{N}_{2}\right)$, the photoemission yield is estimated as $\gamma=10^{-7}$, which is very small. The value of $\gamma$ increases for photons with shorter wavelength. However, those photons are absorbed in air and cannot serve as a nonlocal source of free electrons necessary for positive streamers.

We assume that the photoemission yield is in the huge range of $10^{-7}$ to 1 , and we study the sensitivity of the discharge to its actual value.

\subsection{Initial conditions}

When working with pulsed discharges of a certain repetition frequency, it is important to estimate the density of free electrons (and in general other species) remaining from the previous discharges, as they can influence the propagation of positive streamers. Approximating the weakly curved front by a planar front and assuming that the electric field in the non-ionized region just ahead of the discharge region does not change in time, in zero-dimensional configuration we can estimate the level of the ionization remaining in the repetitive discharge, like in [50]. We set the maximal electric field in the simulated positive discharge to $20 \mathrm{kV} \mathrm{cm}^{-1}$ at $150 \mathrm{mbar}$ (which would correspond to $133 \mathrm{kV} \mathrm{cm}^{-1}$ at 1 bar according to the similarity laws). The electron density where the electric field is maximal scales to $9 \cdot 10^{11} \mathrm{~cm}^{-3}$ at $150 \mathrm{mbar}$ [22], and we used this value as the initial condition for our zerodimensional analysis in the same manner as in [50]. We initialize the densities of the positive ions $\mathrm{N}_{2}^{+}$and $\mathrm{O}_{2}^{+}$such that the plasma is electrically neutral $n_{\mathrm{e}}(0)=n_{\mathrm{N}_{2}^{+}}(0)+n_{\mathrm{O}_{2}^{+}}(0)$. We keep the initial ratio as $n_{\mathrm{N}_{2}^{+}}(0): n_{\mathrm{O}_{2}^{+}}(0)=n_{\mathrm{N}_{2}}(0): n_{\mathrm{O}_{2}}(0)$. The initial densities of all other ions, excited species and groundstate neutrals (except $\mathrm{N}_{2}$ and $\mathrm{O}_{2}$ ) are assumed to be zero. We performed the estimations with ZDPlasKin software [51]. Figure 4 shows the results, and we conclude that the residual electron density in air discharges at 150 mbar with repetition frequency of $1 \mathrm{~Hz}$ is negligible.

We launch a discharge by placing an electrically neutral plasma seed on the axis of the domain at the electrode tip. The maximal electron (and ion) density is $10^{7} \mathrm{~cm}^{-3}$. The decay length of the Gaussian seed is $1 \mathrm{~mm}$, which is smaller than the typical discharge width at 75-150 mbar but comparable with the gap size. The centre of the seed coincides with the electrode tip. This seed was chosen to facilitate the start of the streamer propagation and the chosen parameters do not influence the discharge development. Taking into account the voltage rise time (see figure 2), the seed electrons create a conductive patch around the electrode tip before the voltage becomes high enough and a discharge incepts. After its inception the discharge propagates predominantly due to photoionization.

\section{Experiments}

The stainless steel vessel in our setup is filled with ambient air at pressures between 75-150 mbar. Inside the vessel, the needle anode is placed approximately $18 \mathrm{~cm}$ above a grounded cathode plane. The (grounded) conical holder containing the dielectric rod is placed in the discharge gap, effectively reducing the gap size to $96 \mathrm{~mm}$. The rod is placed directly under the needle, with a gap of approximately $1 \mathrm{~mm}$ between the needle and the (rounded) top of the dielectric rod (see figure 1). The rods used in the experiments were $4 \mathrm{~mm}$ in diameter and protrude approximately $95 \mathrm{~mm}$ from the holder. We used two different rods with a (relative) dielectric permittivity of $\epsilon \sim 4$ (unfilled epoxy resin) and $\sim 8$ (epoxy resin with $\mathrm{TiO}_{2}$ filler).

We apply a positive $19 \mathrm{kV}$ pulse at a repetition frequency of $1 \mathrm{~Hz}$ to the needle in order to generate streamers. Streamer discharges are imaged using a LaVision PicoStar HR12 stroboscopic ICCD camera, which allows stroboscopic gating at $50 \mathrm{MHz}$. This means multiple intensifier gates, $20 \mathrm{~ns}$ apart, can be achieved within a single exposure (and thus a single discharge). By supplying a train of pulses at $50 \mathrm{MHz}$ timed 
to coincide with the start of the discharge to the intensifier, we can study streamer propagation, through stroboscopic imaging, both temporally as well as spatially resolved. This technique is based on the fact that the streamer head (in air) only emits light for a very short time, i.e. effectively only the streamer head emits light $[23,52,53]$. This method was explained in more detail before [7]. Figure 5(a) shows an example of a stroboscopic image at $150 \mathrm{mbar}$ with the epoxy resin rod in the discharge gap. Due to the stroboscopic gating of the intensifier, the image shows maxima and minima in intensity. A semi-transparent white region indicates the area over which this intensity (shown in figure 5(b)) is measured. The measured intensity is filtered using a low pass filter and maxima and minima are detected automatically using a script. From the positions of the maxima and minima the velocity is determined. The result is shown in figure 5(c). We typically distinguish between three distinctive phases. Initially, the inception cloud rapidly expands (1), until it reaches its maximum size and stagnates (2). After this, the inception cloud breaks up into separate streamer channels that propagate at a rather steady velocity (3). This discharge behavior is similar to the behavior observed by Chen et al [19] for experiments in nitrogen with a $20 \%$ admixture of oxygen. It should be noted that the stagnation of the inception cloud may cause maxima and minima in the measured intensity to overlap partially or fully, making their identification impossible. Therefore, the presented velocity in the stagnation phase may in fact be even lower.

\subsection{Discharge morphology}

Experiments were performed at 75 and 150 mbar ambient air. Figure 6 shows an overview of the typical discharge morphologies observed in these experiments. No surface discharges were observed under these conditions. Discharges at $75 \mathrm{mbar}$ (figures 6(a) and (c)) all showed a similar behavior. Initially, the discharge rapidly expands before eventually stagnating. In all of our experiments at $75 \mathrm{mbar}$ a negative streamer propagating upwards from the grounded holder connects with the inception cloud before the inception cloud breaks up into separate channels. The inception cloud was observed to grow to $\sim 55-60 \mathrm{~mm}$ in all cases at $75 \mathrm{mbar}$. This is somewhat lower than the theoretical maximum value of $83 \mathrm{~mm}$, calculated using equation (1) [19].

No differences were observed between the discharge around the unfilled epoxy resin rod (figure 6(a): $\epsilon=4$ ) and around the $\mathrm{TiO}_{2}$ filled rod (figure 6(b): $\epsilon=8$ ). Comparing these cases with the case without the rod, we observe that the rod partially blocks the inception cloud. The rod appears to block a roughly conical section of the inception cloud, which is also observed in simulations (see figure 10). The discharge avoids this conical region and consequently has to travel further before it can reach the cathode. Most likely, this longer path causes the discharges to bridge the discharge gap more slowly when a rod is present.

For 150 mbar (figures 6(d)-(f)) we also observe a rapidly expanding inception cloud that eventually stagnates for both

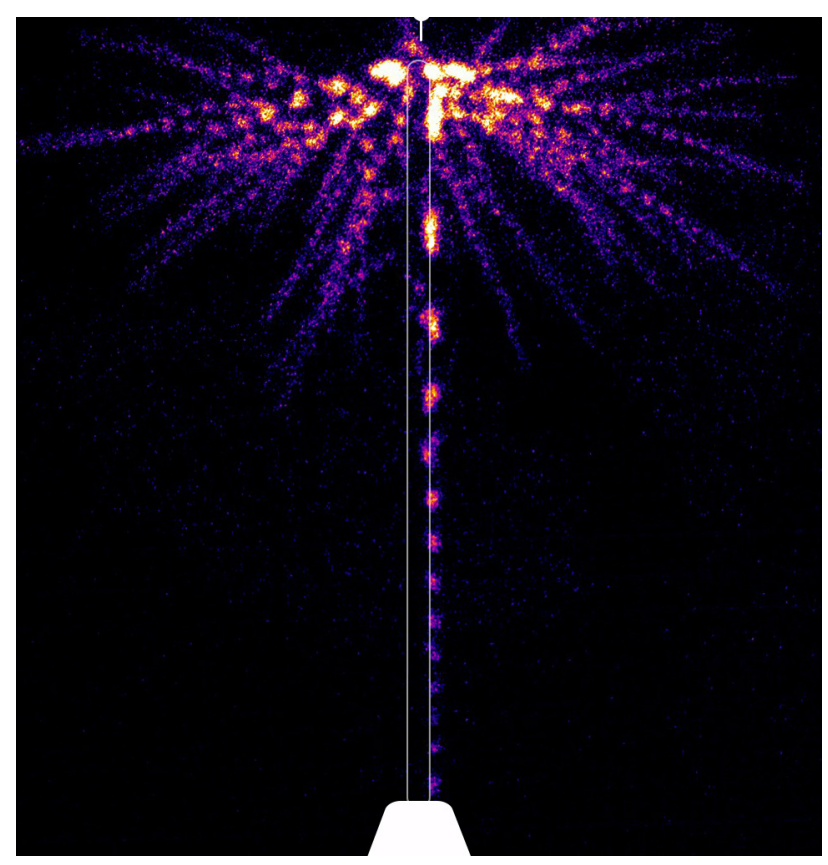

Figure 7. Typical stroboscopic image of a discharge at $600 \mathrm{mbar}$ in air for the rod with $\mathrm{TiO}_{2}$ filler, $\epsilon=8$. A positive surface streamer can be observed and its velocity is clearly much larger than the velocity of the bulk streamers.

with and without a rod. The radius of the inception cloud is roughly halved to $\sim 25-30 \mathrm{~mm}$ (compared to $75 \mathrm{mbar}$ ). This value is again somewhat lower than the theoretical maximum (see equation (1)), and the scaling with pressure is maintained [19]. After stagnating, the inception cloud breaks up into separate channels. No differences were observed between the discharges around the two rods. However, without the rod the streamer channels emerging from the inception cloud are significantly thinner and less numerous. It was also observed that these streamer channels often emerge later or not at all. The absence of a dielectric rod could cause the inception cloud to maintain its spherical shape and prevent the emerge of streamers while the rod breaks the semispherical symmetry. Some streamer channels can be observed that originate from the high voltage feedthrough above the needle position (see upper part of figure 6(f)). It could be possible that these channels trigger the breaking up of the inception cloud. To the best of our knowledge, the propagation velocity of the channels is not influenced by the channels originating from the HV feedthrough. Note that the filaments emerging from the inception cloud cannot be simulated using our 2D model.

\subsection{Effect of pressure}

To investigate the role of pressure, we repeated the experiment for a pressure of $600 \mathrm{mbar}$ and otherwise identical conditions. Due to the increased pressure at unchanged voltage, inception of the discharge was not always observed. For the cases where inception was observed, we always observed a surface streamer. Figure 7 shows a typical stroboscopic image from this experiment. A positive surface streamer can clearly be distinguished here. Moreover, a clear inception cloud cannot 
be observed in this image, possibly due to its reduced size. The close proximity of many discharge filaments also makes it hard to identify the inception cloud in the initial stage of the discharge. As the inception cloud now fits into the gap between the electrode and the rod, the discharge breaks up into filaments within the gap, and streamers can reach the rod surface, see figure 7. We will elaborate on this in section 5 .

The close proximity of many discharge filaments also makes it hard to identify the inception cloud in the initial stage of the discharge. As the inception cloud now fits into the gap between the electrode and the rod, the discharge breaks up into filaments within the gap, and streamers can reach the rod surface, see figure 7. We will elaborate on this in section 5. As can be seen in figure 7, the velocity of the surface streamers is clearly larger than that of the bulk gas streamers. This finding in similar setups has been observed before $[7,9,10,16]$. The discussion on this is beyond the scope of this paper as we focus on the affinity of discharges for dielectric surfaces.

\section{Comparison of simulations with experiments}

Simulations (section 2) and experiments (section 3) are compared in figure $8(\mathrm{c})$ where we show the stroboscopically integrated light emission of a positive discharge.

In simulations, we assume that light emission is proportional to impact ionization $n_{\mathrm{e}} \alpha$ ( $\alpha$ is impact ionization coefficient) and we also integrate it in the viewing direction across the area of the display. The quenching rates were taken from [52, 54, 55]. The limited number of grid cells ultimately restricts how long we can simulate discharge propagation, and therefore the elapsed time is only about one stroboscopic period.

First, we compare the results without the dielectric rod to have a reference case. Then we compare the results with a dielectric rod in air at 150 and 75 mbar. In all the cases at $75-150$ mbar no surface streamers were observed. Both in experiments and simulations, no difference has been observed when the rod with a relative permittivity of 4 was replaced with the rod with a relative permittivity of 8 . It seems that the relative permittivity, at least in this range, does not play a significant role in determining the discharge morphology. We will discuss the role of field enhancement in more detail in section 5.1

Figure 9 shows the calculated and measured inception cloud discharge velocity as a function of distance from the needle. Experimental values were obtained using the method described by figure 5 . In simulations, the velocities were calculated by taking the time derivative of the position of the maximum of the electric field.

Experimentally, no differences were observed between the velocities measured with the unfilled epoxy resin $\operatorname{rod}(\epsilon=4)$, the $\mathrm{TiO}_{2}$-filled rod $(\epsilon=8)$ and without a dielectric rod. For $75 \mathrm{mbar}$, inception cloud velocities of up to $\sim 2 \mathrm{~mm} \mathrm{~ns}^{-1}$ were found for all cases. This velocity decreases to $\sim 0.5 \mathrm{~mm} \mathrm{~ns}^{-1}$ when the inception cloud stagnates. As was mentioned in section 3.1 a negative streamer, originating from the grounded holder, causes the discharge to bridge the gap before the inception cloud breaks up into separate streamer channels.

For 150 mbar, a similar trend was observed, albeit with a smaller inception cloud and lower velocities. Indeed, for the same applied voltage and the same gap, we get a lower $E / N$ ratio which translates into smaller velocities. Figure 5 shows that the differences between the three cases are again very small. Our results show a typical velocity for the inception cloud expansion of $\sim 1 \mathrm{~mm} \mathrm{~ns}^{-1}$. This velocity decreases to $\sim 0.2 \mathrm{~mm} \mathrm{~ns}^{-1}$ before the inception cloud breaks up and the streamers propagate at $\sim 0.4 \mathrm{~mm} \mathrm{~ns}^{-1}$. Note that this part is omitted from figure 9 because we focus on the cylindrically symmetric part of the discharge here.

One would expect the velocity of the streamers emerging from the inception cloud without a rod to be lower than those reported for both rods, as we observed these streamers to be significantly thinner (see figures 6(d)-(f)), but we do not observe such a difference.

The velocities we find here are similar to those reported by Chen et al [19]. They measured velocities for $100 \mathrm{mbar}, 20 \mathrm{kV}$ in a point-plane gap in otherwise similar conditions. Initially, they observed a relatively high velocity $\left(\sim 1 \mathrm{~mm} \mathrm{~ns}^{-1}\right)$ and stagnation of this inception cloud $\left(\sim 0.09 \mathrm{~mm} \mathrm{~ns}^{-1}\right)$ before it breaks up into separate streamer channels and the velocity increases again $\left(\sim 0.36 \mathrm{~mm} \mathrm{~ns}^{-1}\right)$. These velocities are comparable to the velocities we find. It should be noted however that the velocity decrease in [19] is more severe (down to $\sim 0.09 \mathrm{~mm} \mathrm{~ns}^{-1}$ ). Possibly, the stagnation of the inception cloud is more severe in our case as well, but the close proximity of the maxima in intensity in the stroboscopic image do not allow us to resolve this.

Numerically, we get similar results. For $75 \mathrm{mbar}$ and an $\epsilon=4$ dielectric rod, the velocity was found to increase from 0.5 to $0.7 \mathrm{~mm} \mathrm{~ns}^{-1}$ in the first $10 \mathrm{~mm}$ of the discharge. Although somewhat lower than the experimentally determined velocity, the positive trend in the velocity suggests that our simulations are consistent with our experiments. For 150 mbar and an $\epsilon=4$ dielectric rod, the velocity increases from 0.3 to $0.5 \mathrm{~mm} \mathrm{~ns}^{-1}$ in the same stage. These results are in accordance with the experiments in the inception phase of the discharge and with the observations of the bulk discharge in air in $[9,10]$. The velocities slightly increase as the discharge propagates due to the rising voltage.

\section{Discussion}

Contrary to our expectations, we have not observed cylindrically symmetric positive discharges propagating on the surface of a dielectric, neither experimentally nor numerically. Figures 6 and 10 clearly show that the inception cloud avoids the area near the rod. In the simulations, we varied the photoemission yield $\gamma$ from $10^{-7}$ to 1 in order to increase the effect of the dielectric surface. We expected that by supplying more free electrons from the dielectric surface we would be able to increase the affinity of the discharge for the surface. However, the increase of the photoemission yield by seven orders of magnitude was still not enough to make the discharge propagate along the dielectric rod.

We attribute this behavior to the abundance of photoionization in air. In the inception cloud phase, photoionization in air will provide the discharge with an abundance of free electrons. The dielectric rod partially blocks the expansion of the 


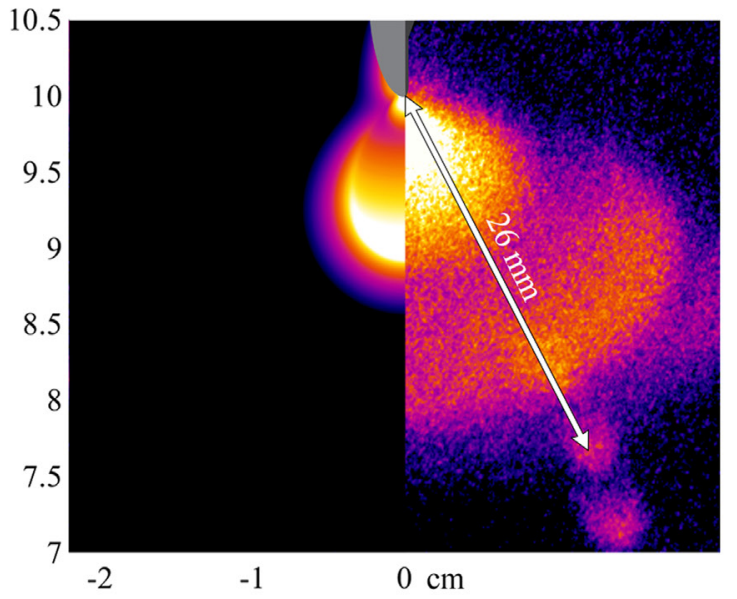

(a)

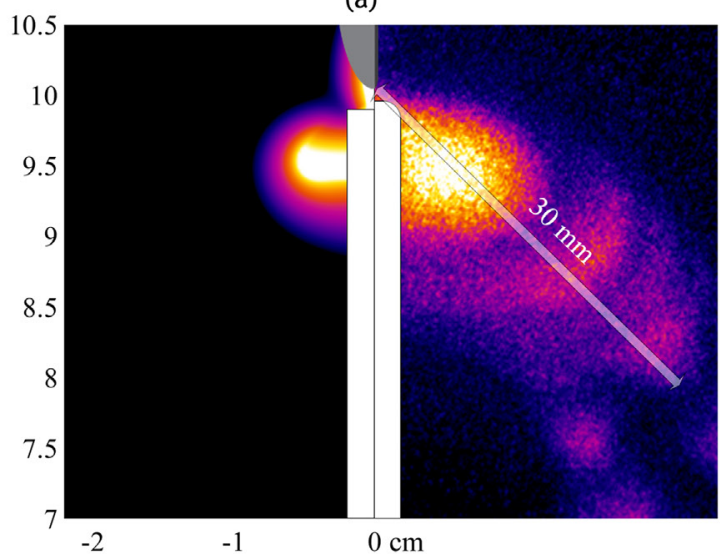

(b)

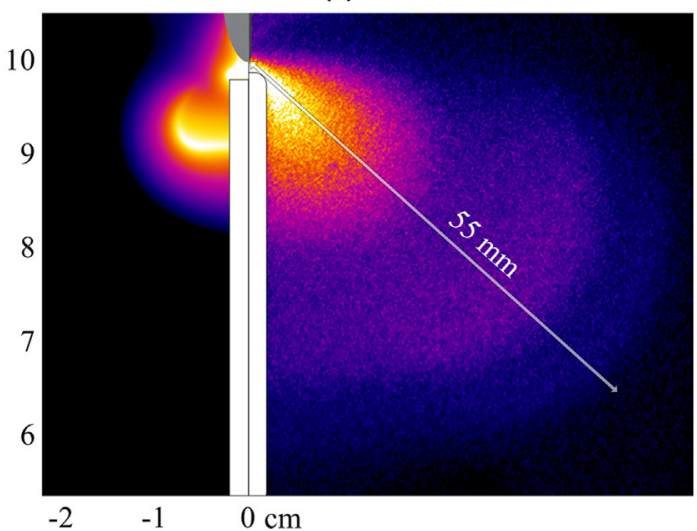

(c)

Figure 8. Light emission of a positive streamer discharge in air simulated (the left half) and observed experimentally with the stroboscopic effect (the right half). We show three cases: (a) at $150 \mathrm{mbar}$ in the absence of the dielectric rod, (b) at 150 mbar with the dielectric rod, (c) at 75 mbar with the dielectric rod. The arrow indicates the observed inception cloud size. The distance between the maxima of the light emission corresponds to $10 \mathrm{~ns}$ of the simulated discharge propagation, and $20 \mathrm{~ns}$ of discharge propagation in the experiments (the stroboscopic ICCD camera operates at 50 $\mathrm{MHz}$ with a $10 \mathrm{~ns}$ on and $10 \mathrm{~ns}$ off time). In the simulations, the elapsed time is about one stroboscopic period. The measured and calculated velocities of the discharge are given in figure 9 .

inception cloud because it blocks photoionization, but it hardly influences its propagation otherwise. As was discussed in section 3.1 , a conical region of the (semi)spherical expanding shell is blocked. Despite the altered shape of the inception cloud, our experimental results suggest that the breaking up of the inception cloud into separate channels takes place at roughly the same distance from the needle as would be the case without the dielectric rod (see figure 6). Any additional free electrons generated by photoemission will not cause the discharge to deviate from its spherical shape. Equation (1) gives a theoretical maximum radius of the inception cloud by assuming the inception cloud to be an ideally conducting sphere [19]. Using $U=20 \mathrm{kV}$ as the applied voltage, we find that the experimentally measured inception cloud size is about $\sim 60-70 \%$ of its theoretical maximum both for 75 mbar and $150 \mathrm{mbar}$, when the Townsend scaling of the breakdown field $E_{c}$ with pressure is used. In the work by Chen et al an actual size of $\sim 90 \%$ of the theoretical maximum size was found. Therefore, this equation provides an upper bound and a good estimate for the inception cloud size.

We expect the rod to roughly block a conical region with a solid angle of $\Omega=2 \pi(1-\cos 2 \theta)$, where $\theta$ is the angle between the line from the electrode tip to the tangent to the rod and the vertical axis if the inception cloud is bigger than the gap between the electrode and the rod. This is depicted in figure 11(a). The presence of the shaded conical region can be explained first by the fact that the dielectric is opaque for the photons providing photoionization. However, the region is larger than what is occupied by the dielectric rod. This can be explained by the electrons produced due to photoionization just outside of the dielectric rod have hardly any space to multiply and create an avalanche that could contribute to the streamer formation. Those electrons are more likely to attach to the surface than to end up in the streamer head (see figure 10 for the potential lines).

As the pressure is increased while the voltage is kept, the inception cloud decreases. If it is still bigger than the rod, the horizontal distance between the inner edge of the cone and the rod will decrease. Additionally, the distance to the grounded cathode will increase. Figures 11(b) and (c) show a schematic representation of this process for lower and higher pressure, respectively. Figure 12 shows theoretical values for the maximal inception cloud radius, the horizontal distance of the inner cone edge to the rod and its vertical distance to the cathode. This figure demonstrates that the reduced size of the inception cloud at higher pressure will cause filaments to emerge from the inception cloud closer to the rod and further away from the cathode. This could make it easier for surface discharges to appear, as the proximity of the filaments to the rod may cause them to encounter it. Filaments have been observed to propagate along dielectric surfaces upon encountering them before [1, 2, 7-10, 14].

To test the hypothesis that a dielectric surface will simply block part of the inception cloud, we compare two PMMA $(\epsilon \approx 3.6)$ rods with diameters of 6 and $10 \mathrm{~mm}$ that were placed at varying distances from the needle. The rods had a flat top and were placed in a grounded holder at a constant distance of approximately $100 \mathrm{~mm}$ from the needle. The same voltage pulse shape (see figure 2) was used. We measured the geometrical angle $\theta$ indicated in figure 11(a) and compared this to the angle $\varphi$ the discharge makes with the vertical axis. Experiments were performed at 75 mbar. An example of such 


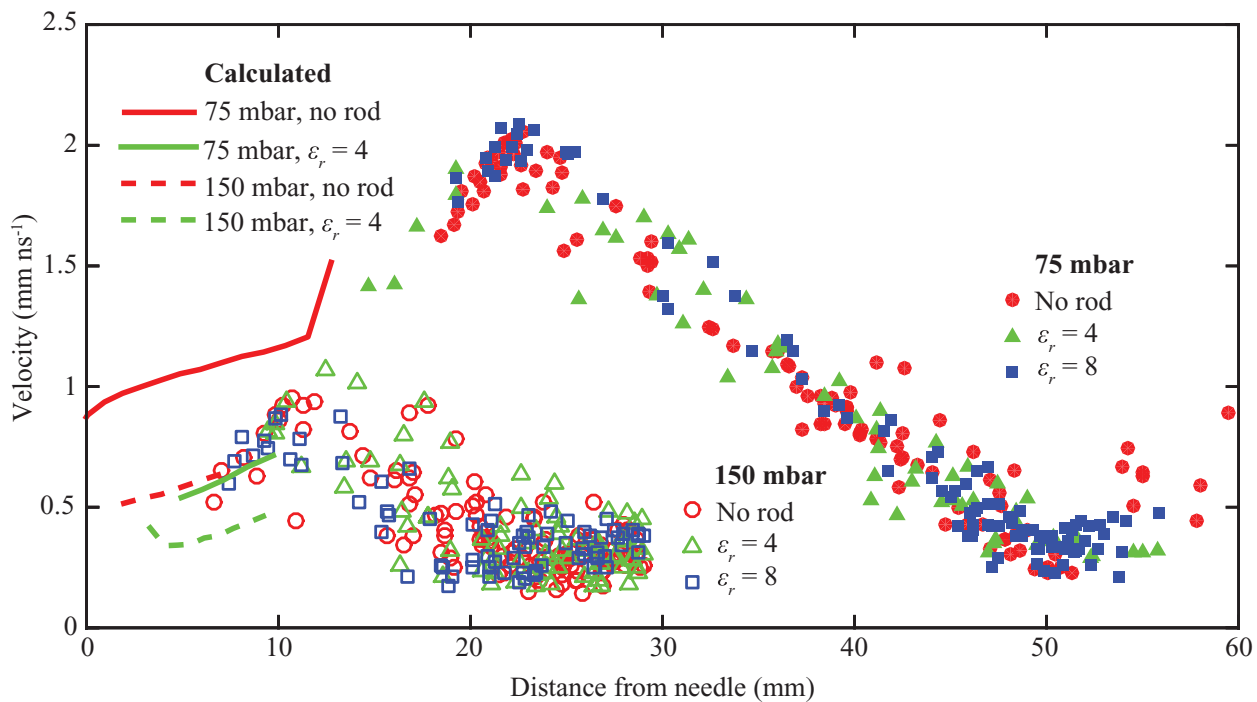

Figure 9. Velocity of the discharge before destabilization into filaments (i.e. in the inception cloud phase) as a function of distance from the needle anode for 75 and $150 \mathrm{mbar}$, for two different rods and without a rod. Numerically calculated values are also shown. The theoretical maximal radii of the inception cloud are $42 \mathrm{~mm}$ at $150 \mathrm{mbar}$ and $84 \mathrm{~mm}$ at $75 \mathrm{mbar}$, according to equation (1).
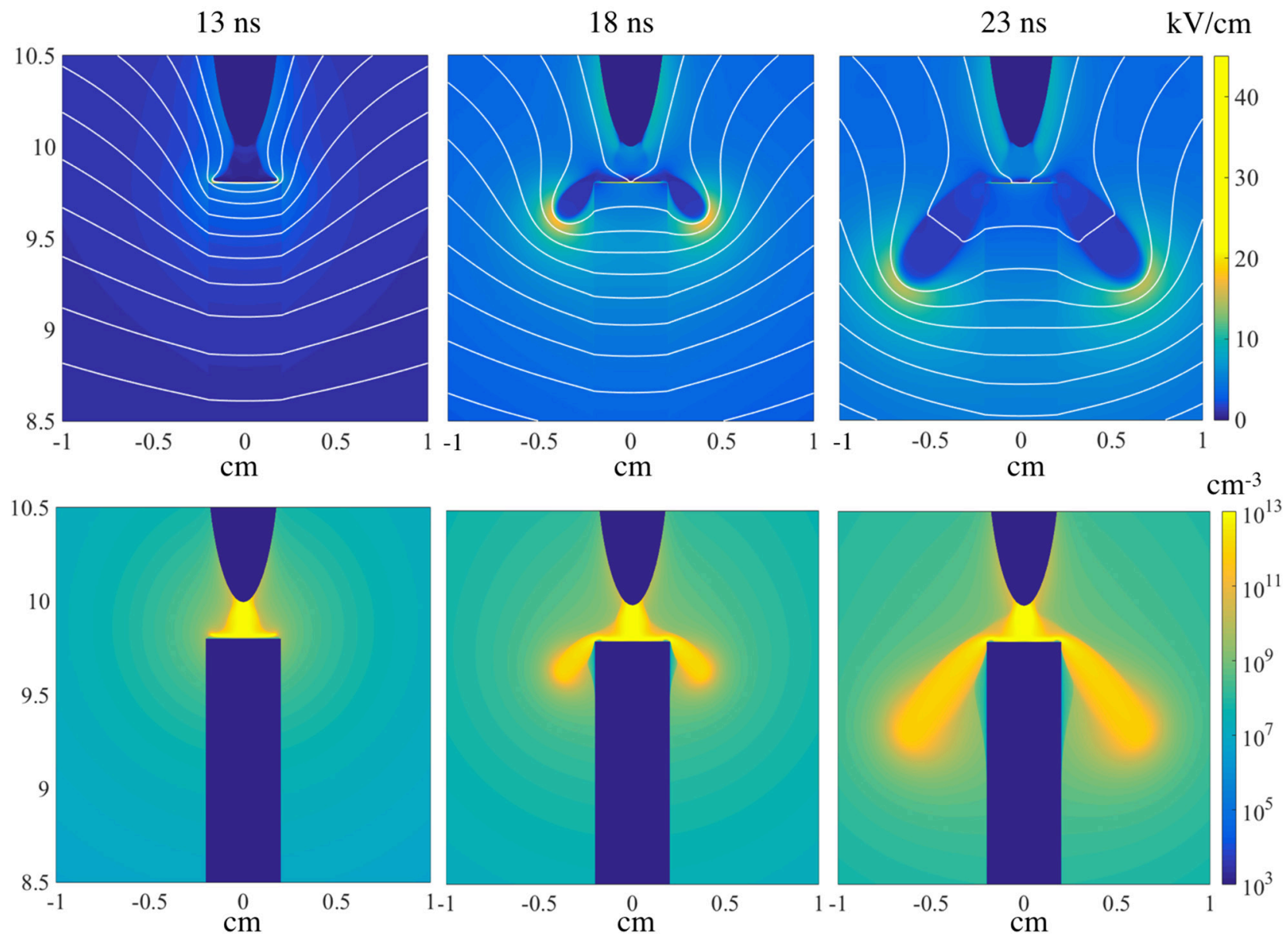

Figure 10. Simulation results zoomed-in into the area of discharge development (see figure 3) in $150 \mathrm{mbar}$ air after $13 \mathrm{~ns}, 18 \mathrm{~ns}$, and $23 \mathrm{~ns}$ from the start of a pulse for $\epsilon=4$ and $\gamma=1$. Despite the large value of the photoelectron yield to maximize the attraction to the rod, but it is still not seen. Upper panel: the absolute value of the electric field in the discharge and the equipotential lines. Lower panel: the electron density on a logarithmic scale.

a measurement is shown in figure 13 . Here, the green line determines the geometrical angle $\theta$ and the black line determines $\varphi$. We define $\varphi$ as the angle between the line from the electrode tip to the center of curvature of the discharge front and the vertical axis. Because we image the discharge stroboscopically, we generally have a few reference points to draw 


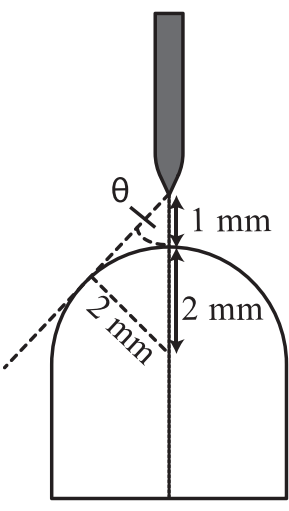

(a)

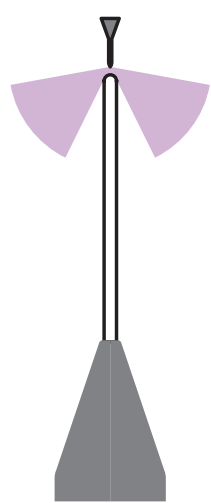

(b)

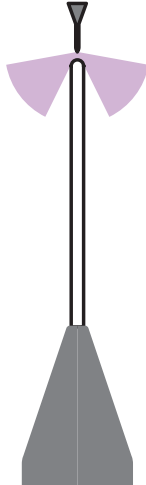

(c)
Figure 11. Schematic drawing showing the geometrical angle $\theta$ between the inception cloud and the vertical axis (a), and the point where the inception cloud breaks up for low (b) and high (c) pressure.

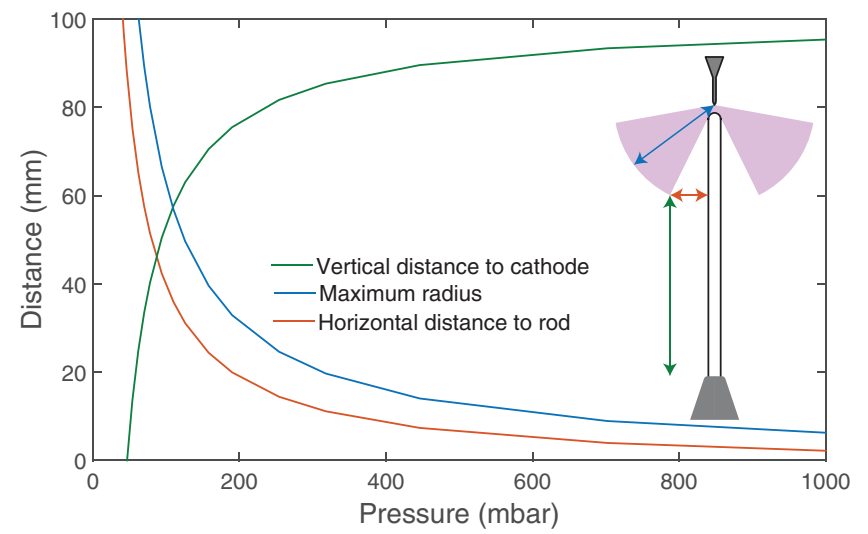

Figure 12. The theoretical size of the inception cloud according to equation (1) (blue), and the radial distance of the cloud cone to the rod (red) and the axial distance of the cloud cone to the cathode (green) at the point where the inception cloud breaks up.

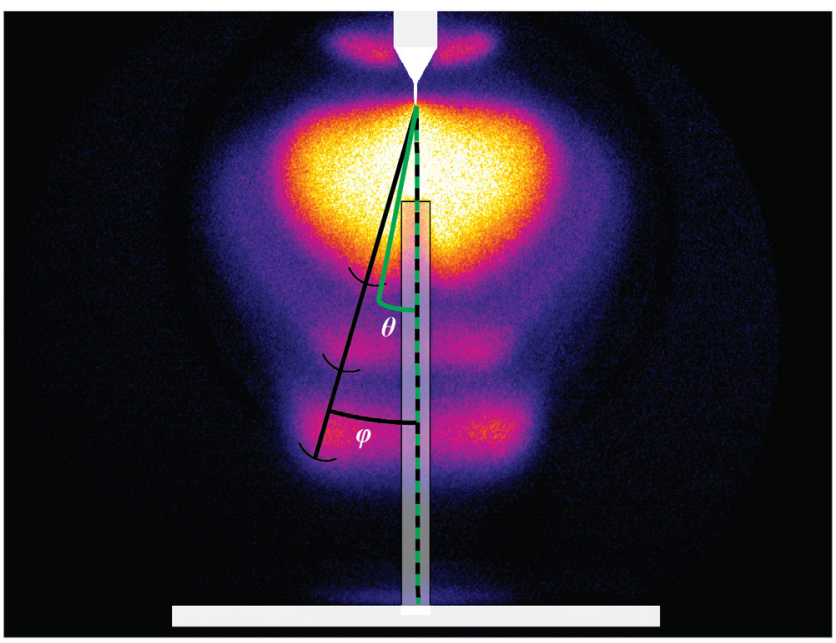

Figure 13. Stroboscopic image of the discharge at $75 \mathrm{mbar}$ with the $6 \mathrm{~mm}$ rod, indicating how the geometrical angle $\theta$ (green) and the discharge angle $\varphi$ (black) are determined.

this line, as can be seen in figure 13. Although $\varphi$ is determined from a 2D-projection of emission from a 3D phenomenon, we expect that the error using this method is still within

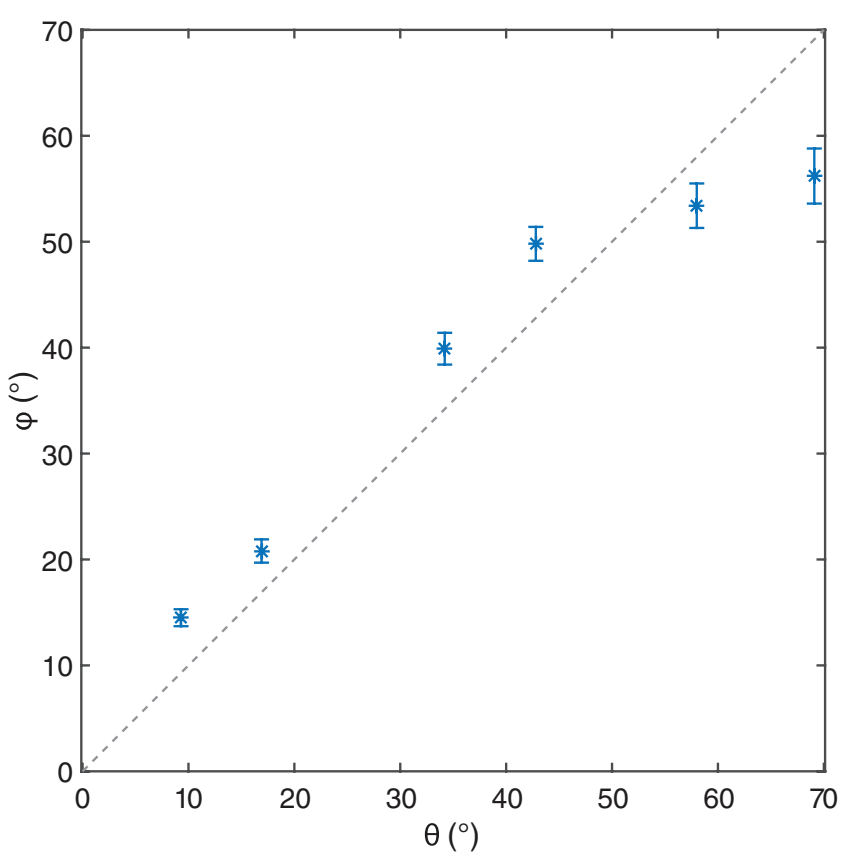

Figure 14. Discharge angle $\varphi$ as a function of the geometrical angle $\theta$. The dotted line shows $\varphi=\theta$, which would be the case if discharge propagation would be governed by the geometrical angle only.

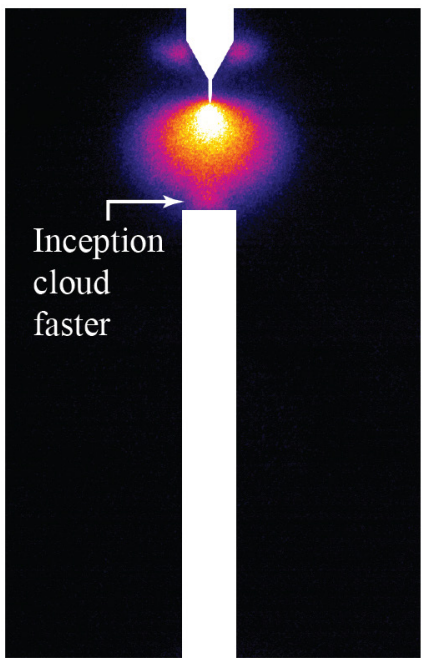

a)

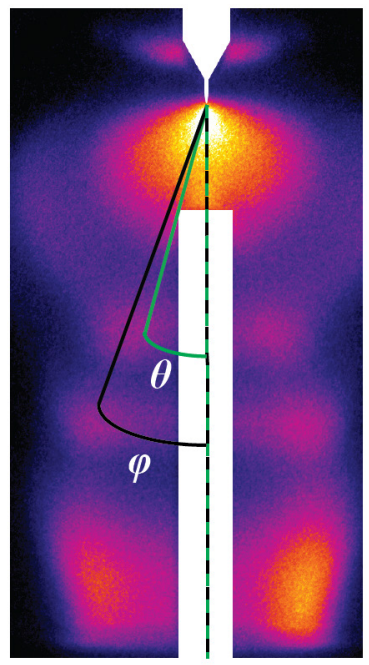

b)
Figure 15. When the rod is placed further from the needle, the inception cloud shape deviates from its spherical shape due to the field enhancement caused by the dielectric (a), only for this larger gap. As a result, the discharge propagates at an angle larger than $\theta$ (b).

reasonable bounds. Nevertheless, it should be taken into account that $\varphi$ could be consequently underestimated using this method. Both rods were placed at three different distances from the needle in order to compare six different values for $\theta$. Figure 14 shows the observed discharge angle $\varphi$ as a function of the geometrical angle $\theta$. As can be seen, $\varphi$ clearly increases with $\theta$, as was expected. We find that the discharge propagates at an angle that is somewhat larger than $\theta$, except for large values of $\theta$.

To analyze this behavior, we investigated the inception cloud size at the moment it reaches the top of the rod. 


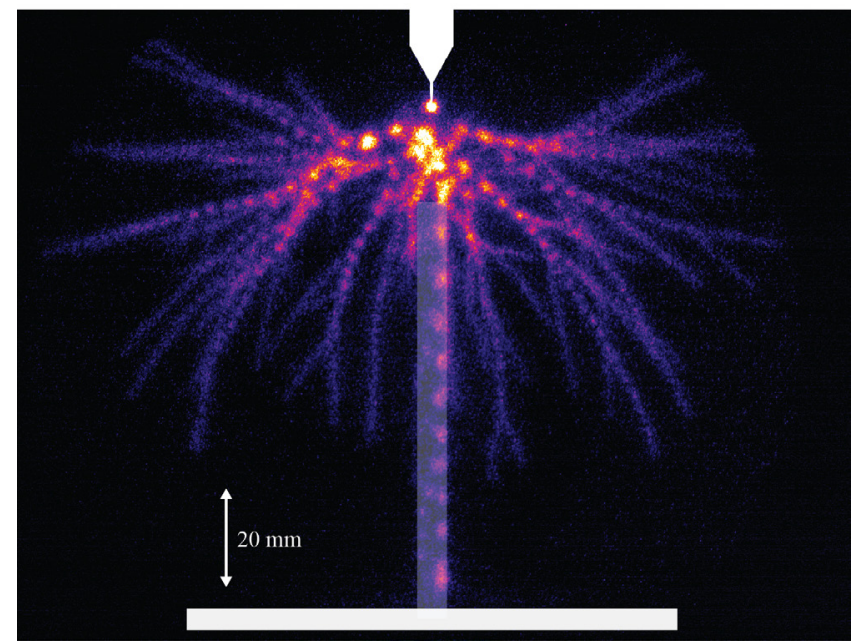

Figure 16. Example of a surface discharge at 400 mbar with the $6 \mathrm{~mm}$ PMMA rod placed in the discharge gap.

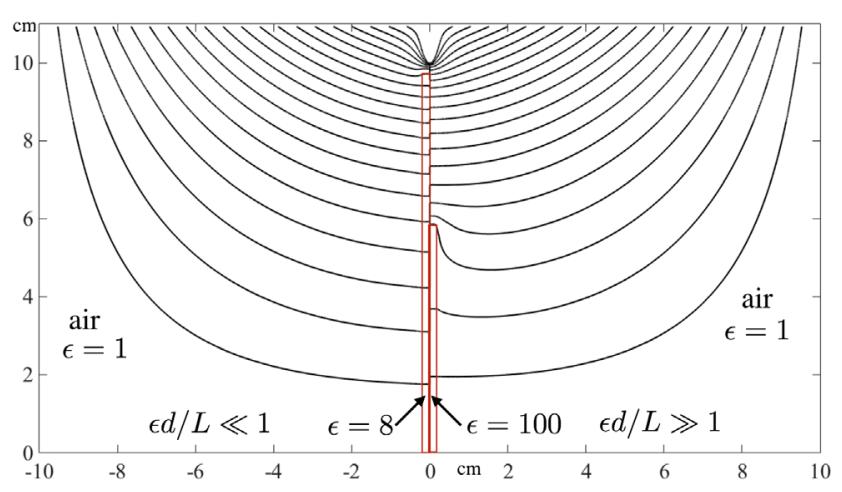

Figure 17. Equipotential lines in the setup for two cases $\epsilon d / L \ll 1$ and $\epsilon d / L \gg 1$. In the left half of the figure, $\epsilon=8, d=2 \mathrm{~mm}$, and $L=98 \mathrm{~mm}$, as used in our experiments and simulations. In the right half of the figure, $\epsilon=100, d=42 \mathrm{~mm}$, and $L=58 \mathrm{~mm}$. Clearly, in the latter case a discharge is more predisposed to be attracted by the dielectric rod.

The dielectric rod will cause a field enhancement between the discharge and the rod. This field enhancement causes the inception cloud to deviate slightly from its spherical shape (see figure 15(a)). Due to the deviation in the inception cloud shape, it is possible that the discharge will propagate at a slightly larger angle $\varphi$. The deviation in inception cloud shape is shown in figure 15(b). For radii much larger than the rod radius, we expect the discharge to propagate at $\varphi<\theta$ because the background electric field points mostly downwards (towards the cathode), rather than radially outwards.

As was mentioned before, we expect to observe more surface discharges when the inception cloud breaks up close to the rod. As figure 12 shows, high pressure is expected to increase the likeliness of surface discharges. The vertical position of the rod with respect to the needle was expected to be important as well, as placing the rod further from the needle decreases the geometrical angle $\theta$.

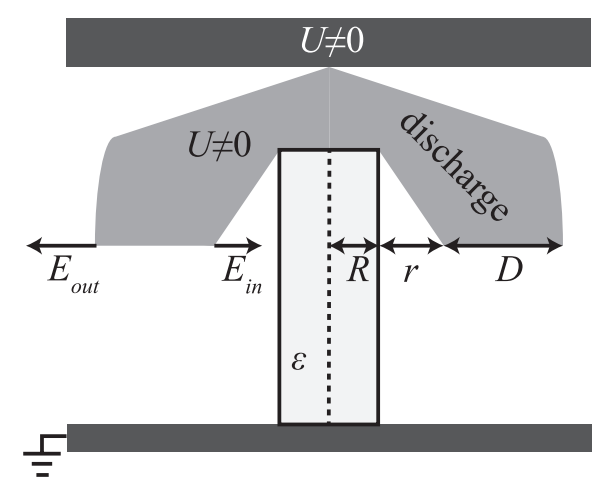

(a)

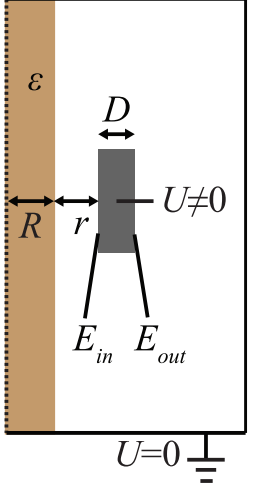

(b)
Figure 18. Schematic drawing showing the relevant parameters at play when discussing field enhancement in the presence of a discharge. A streamer discharge as depicted in the left figure is approximated as a perfectly conductive ring under a fixed potential as shown in the right figure.

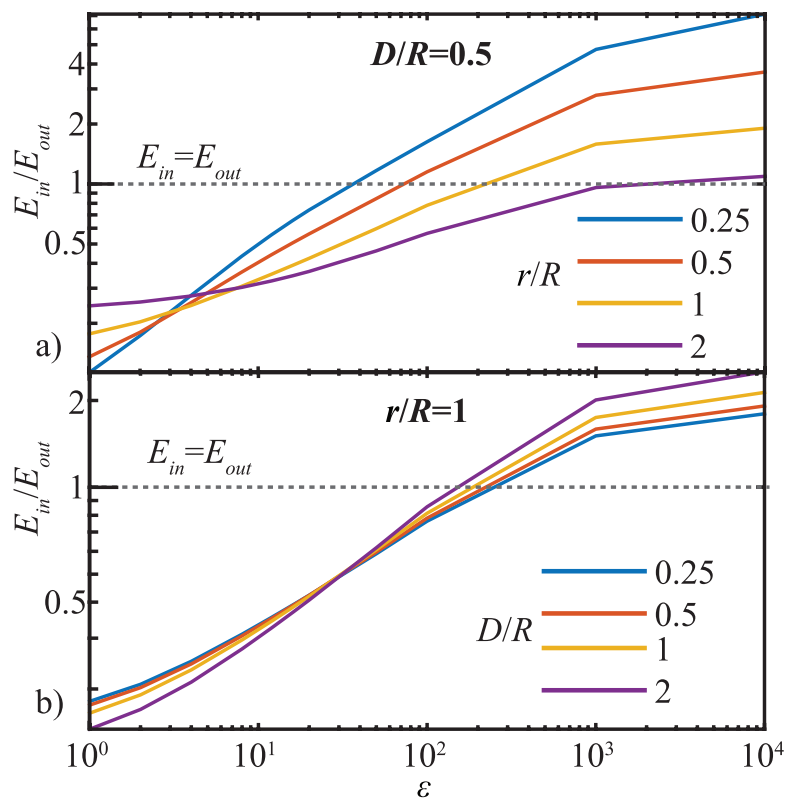

Figure 19. Ratio between the radial field on the inside of a conducting ring at fixed potential $E_{\text {in }}$ and the field on the outside of the ring $E_{\text {out }}$ as a function of relative permittivity of the dielectric $\operatorname{rod} \epsilon$ for varying distance to the rod $r / R$ (a) and for varying ring thickness $D / R$ (b). The rod thickness $R$ was kept constant in all simulations. The dotted line shows the conditions when $E_{\mathrm{in}}=E_{\text {out }}$. There exists a threshold $\epsilon_{\mathrm{th}}$ such, that for $\epsilon>\epsilon_{\mathrm{th}}, E_{\mathrm{in}}>E_{\mathrm{out}}$ meaning that the electrostatic attraction of the conductive ring to the dielectric rod dominates its self-repulsion.

As a result, the inception cloud will break up closer to the rod, and the discharge is more likely to propagate along the dielectric surface. Our results suggest that this hypothesis is correct: we only observe surface discharges for relatively high pressure and small $\theta$. Figure 16 shows an example of such a surface discharge at 400 mbar. It is hard to distinguish an inception cloud in this case. Equation (1) predicts a maximum radius of $\sim 1.5 \mathrm{~mm}$ and we have observed actual radii up to $\sim 0.7 R_{\max }$ before. This is smaller than the gap $(\sim 20 \mathrm{~mm})$ 
in this case, meaning that the inception cloud breaks up before the discharge reaches the rod. It is important to note that the broken symmetry implies that our 2D cylindrical simulation model cannot be used to study these surface discharges.

\subsection{Field enhancement in the absence of a discharge: geometrical effect}

Commonly discharges follow the electric fields lines, and therefore let us study the equipotential lines in our setup. In figure 17, we compare the case with $\epsilon=8$, the gap between the dielectric rod and the electrode $d=2 \mathrm{~mm}$, the length of the $\operatorname{rod} L=98 \mathrm{~mm}$, and the case with $\epsilon=100, d=42 \mathrm{~mm}$, and $L=58 \mathrm{~mm}$. It is clear that the potential lines look drastically different. In the latter case a discharge will be more predisposed to be attracted to the dielectric rod as the electric field points to the sides of the rod.

To understand the role that the geometric parameters play in modifying the background field, let us consider a simple model. In a plate-to-plate electrode geometry with a potential difference $\Delta V$ a part of the gap of length $L$ is filled by a dielectric with the dielectric constant $\epsilon$ and the other part is an air gap of distance $d$. The electric field inside the dielectric $E_{\epsilon}$ can be easily calculated and is given by

$$
E_{\epsilon}=\frac{\Delta V}{L} \frac{1}{1+\epsilon d / L}
$$

If $\epsilon d / L \ll 1$, the field inside the dielectric is essentially independent of $\epsilon$. If $\epsilon d / L \gg 1$, then $E_{\epsilon}$ is small and the potential drop is compressed in the air gap and the electric field there is large. Based on this simple model, we take the ratio $\epsilon d / L \ll 1$ as an indicator of the electric field around the dielectric.

When $\epsilon d / L \ll 1$, the dielectric hardly modifies the background electric field, which is true for the case on the left side of figure 17, whereas when $\epsilon d / L \gg 1$, the electric field is severely perturbed. Essentially, in our parameter regime when a dielectric rod fills almost all the gap between the needle and the cathode and the dielectric constant of the rod is not very high, the field enhancement next to the dielectric rod is initially negligible.

\subsection{Field enhancement in the presence of a discharge}

In the presence of a discharge, the local electric field is modified by the space charge in the streamer. To understand the forces acting on the cylindrical streamer let us introduce a simple approximation. We approximate a cylindrical streamer head with its net electric charge as a perfectly conductive ring at a fixed potential $U \neq 0$. We assume that the distance between the streamer head and the rod (that fills all the gap) is $d$, and $D$ is the size of the streamer head. The walls around the streamer are grounded (the rod touches them) and set far away so that the boundary conditions on the walls do no influence the field around the streamer head. The setup is shown in figure 18 .

Using the modeling toolkit Plasimo [56] we numerically calculate the electric field in setup figure 18. We compare the radial component of the field on the inside of the streamer ring $E_{\text {in }}$ with the radial component of the field on the outside of this ring $E_{\text {out }}$. We vary the thickness $D$ of this ring (defined as the outer radius minus the inner radius), the distance to the dielectric rod $r$ and the relative permittivity $\epsilon$ of the dielectric rod. The geometry and the results of our simulations are presented figure 19 . We find that for every set of parameters there exists a threshold dielectric constant $\epsilon_{\text {th }}$ for which $E_{\text {in }}>E_{\text {out }}$. That means that the electrostatic attraction of the conductive ring to the dielectric rod dominates the electrostatic selfrepulsion of the ring. The actual number of $\epsilon_{\mathrm{th}}$ that we obtain in these simple calculations may differ from $\epsilon_{\mathrm{th}}$ in real experiments and full time-dependent simulations. The existence of $\epsilon_{\text {th }}$ clearly illustrates however the competition of two important electrostatic effects. In our cylindrically symmetric simulations (and corresponding experiments), the dielectric permittivity of the rod is obviously not sufficiently large to counteract the self-repulsion of the cylindrical streamer.

In full 3D after the destabilization into filaments, the electrostatic self-repulsion is absent and therefore a streamer is attracted to the dielectric rod, as seen at high pressures (figure 16).

\section{Conclusions}

We have studied interaction of a pulsed positive discharge with a dielectric rod in air in a setup with cylindrical symmetry. Both in experiments and in simulations, no surface discharges have been observed as long as the cylindrical symmetry was preserved, i.e. in the inception cloud phase of the discharge. To our surprise, a positive discharge with cylindrical symmetry tends to move away from the dielectric rod creating a shaded conical region in the centre.

As discussed in section 5.1, for small dielectric permittivities and thin rods, near the dielectric rod the background electric field is hardly perturbed (see left half of figure 17). Therefore, in our geometry there is initially little attraction to the side of the dielectric rod.

When a positive inception cloud emerges that is larger than the gap between the electrode and the rod, it extends beyond the rod edge. This leads to a shading effect with an approximately conical shape. If the inception cloud is smaller than the gap (for example at higher pressures or in larger gaps), the cloud breaks up within the gap, breaking the cylindrical symmetry and streamer filaments are formed already in the gap. The interaction of the inception cloud with the rod is different from the interaction of one of the streamer filaments of the discharge with the rod. As elaborated in section 5.2, for a cylindrically symmetric discharge with a diameter sufficiently larger than the rod diameter, the electric self-repulsion of the discharge tip acts against the electric attraction towards the rod, whereas for non-symmetrical streamer filaments such self-repulsion is absent.

In general, pulsed positive discharge propagation is governed by two dominant mechanisms: free electron supply and electrostatic field effects. Since in air photoionization is abundant, electrostatic field effects largely determine discharge propagation. Furthermore, electron supply to the streamer head near the rod is partly suppressed, since no electron avalanches can approach the streamer head from the rod interior. This effect might be counteracted by 


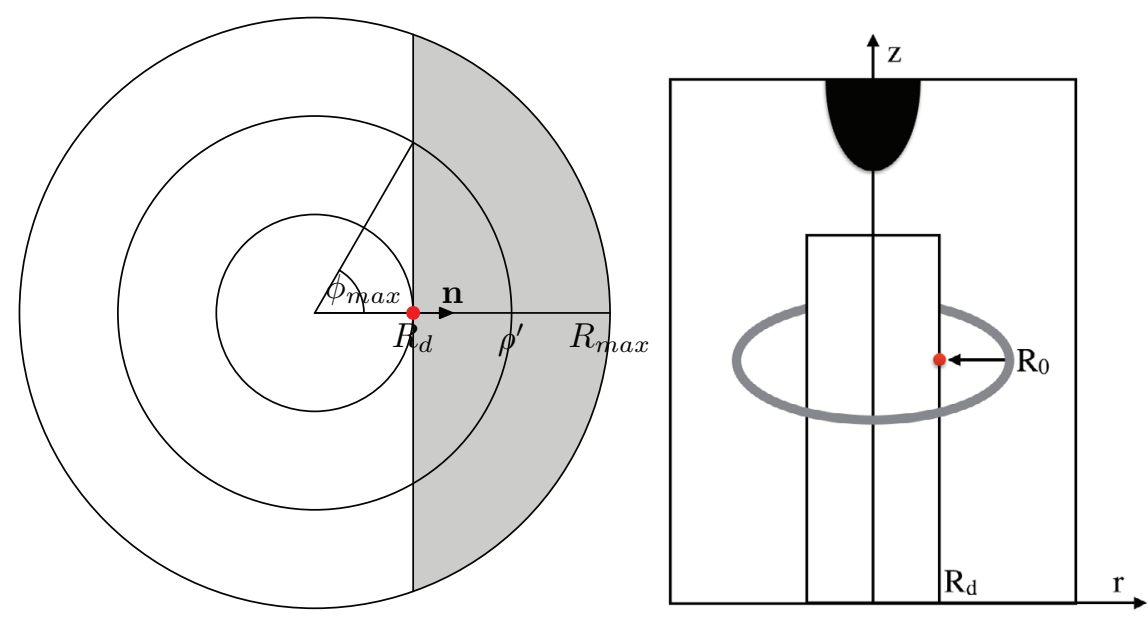

Figure A1. Transverse (left) and axial (right) cross-sections of a cylindrical domain with a cylindrical dielectric rod in the middle. Red color indicates the point of observation on the dielectric cylinder. Grey color indicates the area where a source contributes to the emission from the point of observation.

photoemission from the dielectric surface. The influence of the photoionization, abundant in air, appears to be very strong and dominates over photoemission. Variation of the photoemission yield by seven orders of magnitude from $10^{-7}$ to the unrealistically large value of 1 in our model does not change the discharge behavior. In pure gases like nitrogen, photoionization is suppressed due to the lack of oxygen. Therefore, we expect the dielectric surface to become the dominant source of free electrons, forcing positive streamers to propagate on the surface, even if they have to deviate from the electric field lines. We have indeed observed this behavior and will report about this in our future publication.

Finally, we expect that all our experiments and simulations, which were performed at moderate pressures (75-600 mbar), will give the same results at other pressures assuming that all the lengths scales in the setup (including the size of the dielectric rod) are rescaled according to the Townsend scaling of the discharge. The fact that the quenching factor for photoionization and photoemission do not scale with pressure will hardly change the results.

\section{Acknowledgments}

AD and DT are supported by STW-project 12119, partly funded by $\mathrm{ABB}$, Corporate Research. The authors thank S Pancheshnyi for useful discussions on photoemission, $\mathrm{J}$ Teunissen for discussions of field configurations and D Mihailova for help with building the Plasimo model.

\section{Appendix A. Implementation of the photoemission from a dielectric rod}

The source of photons at any point is assumed proportional to the ionization rate. The photon flux $F$ onto a surface point $\mathbf{r}$ can be obtained by integrating $P(\mathbf{r})$ in equation (12) over the whole volume [57, 58]:

$$
F(\mathbf{r})=\int \frac{P\left(\mathbf{r}^{\prime}\right)}{4 \pi\left|\mathbf{r}-\mathbf{r}^{\prime}\right|^{2}} \frac{\mathbf{n} \cdot\left(\mathbf{r}-\mathbf{r}^{\prime}\right)}{\left|\mathbf{r}-\mathbf{r}^{\prime}\right|} \mathrm{d}^{3} \mathbf{r}^{\prime},
$$

where the second factor under the integral sign in equation (A.1) stands for the cosine of the incidence angle between the vector $\mathbf{r}-\mathbf{r}^{\prime}$ and the vector $\mathbf{n}$ normal the surface at point $\mathbf{r}$.

The integral (A.1) can be calculated directly. The direct methods are used in references [59, 60]. The direct integration can be computationally costly. Moreover, due the shading effect by the dielectric rod (which is assumed opaque for those photons), the number of photons reaching a point on the surface is smaller than it would be in the absence of the rod. The shading effect is geometry dependent and makes the integral in equation (A.1) non-trivial to calculate.

Now let us consider a cylindrically symmetric domain with a cylindrical dielectric rod located on the domain axis. Then we have

$$
\mathbf{r}=\rho \cos \phi \mathbf{e}_{\mathrm{x}}+\rho \sin \phi \mathbf{e}_{\mathrm{y}}+z \mathbf{e}_{\mathrm{z}},
$$

where $\mathbf{e}_{\mathrm{x}}, \mathbf{e}_{\mathrm{y}}$ and $\mathbf{e}_{\mathrm{z}}$ are unit vectors in $x, y$ and $z$ directions, respectively. Due to the cylindrical symmetry we can take the point of observation at $\phi=0$. The normal vector of dielectric cylinder is given by $\mathbf{n}=\mathbf{e}_{\mathrm{x}}$. Then, the integral (A.1) for a photon flux onto the side of a cylinder can be rewritten as

$$
\begin{gathered}
F\left(R_{\mathrm{d}}, z\right)=\int_{0}^{z_{\max }} F_{\text {temp }} \mathrm{d} z^{\prime}, \\
F_{\text {temp }}=\int_{R_{\mathrm{d}}}^{R_{\max }} P\left(\rho^{\prime}, z^{\prime}\right) G\left(\rho^{\prime}, \delta z^{\prime}\right) \rho^{\prime} \mathrm{d} \rho^{\prime},
\end{gathered}
$$

where $z_{\max }$ and $R_{\max }$ stand for the size of the simulation domain, $R_{\mathrm{d}}$ is the radius of the dielectric cylinder, $\delta z^{\prime}=z-z^{\prime}$, and $G\left(\rho^{\prime}, \delta z^{\prime}\right)$ is defined as

$$
\begin{aligned}
& G\left(\rho^{\prime}, \delta z^{\prime}\right)=\frac{1}{2 \pi} \\
& \times \int_{0}^{\phi_{\max }} \frac{\left(\rho^{\prime} \cos \phi^{\prime}-R_{\mathrm{d}}\right) \mathrm{d} \phi^{\prime}}{\left[R_{\mathrm{d}}^{2}+\rho^{\prime 2}-2 R_{\mathrm{d}} \rho^{\prime} \cos \phi^{\prime}+\delta z^{\prime 2}\right]^{\frac{3}{2}}} .
\end{aligned}
$$


Some computational speedup can be achieved by precalculating the inner integral with respect to $\phi$, storing the matrix, and performing summation only for the points on the dielectric cylinder, as done for example in [38]. The maximal angle $\phi_{\max }=\arccos \left(R_{\mathrm{d}} / \rho^{\prime}\right)$ accounts for the shading effect. As it can be seen in figure A1, only photons from the grey area can reach the point (in red) on the surface.

\section{Appendix B. The numerical schemes used in the model}

In our model, the transport equations and the Poisson equation were discretized using a method of finite volumes. The spatial discretization is based on an upwind-biased scheme with the flux limited by the Koren limiter function [61]. This conserves mass and guarantees monotone solutions. The resulting sparse matrix for the Poisson equation was inverted with the numerical package MUMPS [62].

In the coupling of the Poisson equation with the transport equations the electric field is updated at every time step and its values are passed to the transport equations. We solve the system of the coupled partial differential equations with an explicit time integration scheme, namely a two-stage RungeKutta method, which is second-order accurate. This is in line with the accuracy of the spatial discretization.

The time step $\delta t$ varies and it is restricted by the CourantFriedrichs-Levy (CFL) criterion, dielectric relaxation and ionization time scales. The time step is chosen in accordance with [63]. The CFL condition with the advection and diffusion parts combined is necessary for convergence of the equation (3), which we solve numerically by the method of finite volumes. It reads

$$
\delta t \leqslant 0.5 \times \min \left[\frac{\mu_{\mathrm{e}} E_{\mathrm{r}}}{h_{\mathrm{r}}}+\frac{\mu_{\mathrm{e}} E_{\mathrm{z}}}{h_{\mathrm{z}}}+\frac{D_{\mathrm{e}}}{h_{\mathrm{r}}^{2}}+\frac{D_{\mathrm{e}}}{h_{\mathrm{z}}^{2}}\right]^{-1},
$$

where $E_{\mathrm{r}, \mathrm{z}}$ are the radial and the axial components of the electric field, $h_{\mathrm{r}, \mathrm{z}}$ are the sizes of the radial and axial grid cells. The dielectric relaxation time restriction

$$
\delta t \leqslant 0.5 \frac{\epsilon_{0}}{e \mu_{\mathrm{e}} \max \left(n_{\mathrm{e}}\right)}
$$

guarantees that the change of the electric field due to the electric current at every time step is no larger than the electric field. Finally, the ionization time step restriction $\delta t<0.1 / \max \left(\alpha \mu_{\mathrm{e}} E\right)$ is used to accurately resolve the growth of the electron density, in particular in the streamer head. In the simulations the time step varied from about $10^{-13} \mathrm{~s}$ to $10^{-12} \mathrm{~s}$.

\section{References}

[1] Sobota A, van Veldhuizen E V and Stoffels W W 2008 IEEE Trans. Plasma Sci. 36912

[2] Sobota A, Lebouvier A, Kramer N, van Veldhuizen E, Stoffels W, Manders F and Haverlag M 2009 J. Phys. D: Appl. Phys. 42015211
[3] Niemeyer L 1995 IEEE Trans. Dielectr. Electr. Insul. 2 510-28

[4] Yordanov V, Blagoev A, Ivanova-Stanik I, van Veldhuizen E, Nijdam S, van Dijk J and van Der Mullen J 2008 J. Phys. D: Appl. Phys. 41215208

[5] Christen T, Böhme H, Pedersen A and Blaszczyk A 2012 Scientific Computing in Electrical Engineering (New York: Springer) pp 173-81

[6] Mauseth F, Jørstad J and Pedersen A 2012 Annual Report Conf. on Electrical Insulation and Dielectric Phenomena (IEEE) pp 739-2

[7] Trienekens D J, Nijdam S and Ebert U 2014 IEEE Trans. Plasma Sci. 42 2400-1

[8] Jorgenson R E, Warne L K, Neuber A A, Krile J, Dickens J and Krompholz H G 2003 Sandia Report SAND2000-3044

[9] Allen N and Mikropoulos P 1999 IEEE Trans. Dielectr. Electr. Insul. $6357-62$

[10] Meng X, Mei H, Chen C, Wang L, Guan Z and Zhou J 2015 IEEE Trans. Dielectr. Electr. Insul. 22 1193-203

[11] Morales K P, Krile J T, Neuber A A and Krompholz H G 2006 IEEE Trans. Dielectr. Electr. Insul. 13 803-9

[12] Morales K, Krile J, Neuber A and Krompholz H 2007 IEEE Trans. Dielectr. Electr. Insul. 14774

[13] Krile J, Edmiston G, Morales K, Neuber A, Krompholz H and Kristiansen M 2006 Laser Phys. 16 194-201

[14] Voeten S 2013 Matching high voltage pulsed power technologies PhD Thesis Technische Universiteit Eindhoven

[15] Akyuz M, Gao L, Cooray V, Gustavsson T, Gubanski S and Larsson A 2001 IEEE Trans. Dielectr. Electr. Insul. 8 902-10

[16] Laity G, Neuber A, Fierro A, Dickens J and Hatfield L 2011 IEEE Trans. Dielectr. Electr. Insul. 18 946-53

[17] Dubinova A, Trienekens D, Ebert U and Nijdam S 2015 Affinity of pulsed positive discharges to dielectrics in $\mathrm{N}_{2}$ : $\mathrm{O}_{2}$ mixtures Proc. Int. Conf. on Phenomena in Ionized Gases 2015

[18] Briels T M P, van Veldhuizen E M and Ebert U 2008 J. Phys. D: Appl. Phys. $\mathbf{4 1} 234008$

[19] Chen S, Heijmans L C J, Zeng R, Nijdam S and Ebert U 2015 J. Phys. D: Appl. Phys. 48175201

[20] Teunissen J and Ebert U 2016 Plasma Sources Sci. Technol. 25 044005

[21] Ebert U, Nijdam S, Li C, Luque A, Briels T and van Veldhuizen E 2010 J. Geophys. Res. 115 A00E43

[22] Naidis G 2009 Phys. Rev. E 79057401

[23] Briels T, Kos J, Winands G, van Veldhuizen E and Ebert U 2008 J. Phys. D: Appl. Phys. 41234004

[24] Georghiou G, Morrow R and Metaxas A 2001 J. Phys. D: Appl. Phys. 34200

[25] Fujihira M and Inokuchi H 1972 Chem. Phys. Lett. $17554-6$

[26] Tom J, Verhaart H, Verhage A and Vos C 1986 Proc. 2nd Int. Conf. on Conduction and Breakdown in Solid Dielectrics (Erlangen) pp 301-7

[27] Hallac A, Georghiou G and Metaxas A 2003 J. Phys. D: Appl. Phys. 362498

[28] Luque A and Ebert U 2012 J. Comput. Phys. 231 904-18

[29] Hagelaar G J M and Pitchford L C 2005 Plasma Sources Sci. Technol. 14 722-33

[30] Bronold F X and Fehske H 2015 Phys. Rev. Lett. 115225001

[31] Celestin S, Bonaventura Z, Zeghondy B, Bourdon A and Ségur P 2009 J. Phys. D: Appl. Phys. 42065203

[32] Liu X D, Fedkiw R P and Kang M 2000 J. Comput. Phys. $160151-78$

[33] Zheleznyak M, Mnatsakanian A K and Sizykh S 1982 Teplofiz. Vys. Temp. 20 423-8 
[34] Luque A, Ebert U, Montijn C and Hundsdorfer W 2007 Appl. Phys. Lett. 90081501

[35] Jehl A, Farges T and Blanc E 2013 J. Geophys. Res. Space Phys. 118 454-61

[36] Pancheshnyi S, Nudnova M and Starikovskii A 2005 Phys. Rev. E 71016407

[37] Pancheshnyi S, Starikovskaia S and Starikovskii A Y 2000 Chem. Phys. 262 349-57

[38] Babaeva N Y, Bhoj A N and Kushner M J 2006 Plasma Sources Sci. Technol. 15591

[39] Jánský J, Tholin F, Bonaventura Z and Bourdon A 2010 J. Phys. D: Appl. Phys. $\mathbf{4 3} 395201$

[40] Capeillere J, Ségur P, Bourdon A, Célestin S and Pancheshnyi S 2008 J. Phys. D: Appl. Phys. 41234018

[41] Phelps A and Petrovic Z L 1999 Plasma Sources Sci. Technol. 8 R21

[42] Bokhan P and Zakrevsky D E 2013 Phys. Rev. E 88013105

[43] Jaksts A and Cross J 1981 Can. Electr. Eng. J. 6 14-8

[44] Griffith O, Holmbo D, Habliston D and Nadakavukaren K 1981 Ultramicroscopy 6 149-56

[45] Dam R J, Burke C A and Griffith O H 1974 Biophys. J. 14 467-72

[46] Fujihira M, Hirooka T and Inokuchi H 1973 Chem. Phys. Lett. 19 584-87

[47] Buzulutskov A, Breskin A and Chechik R 1997 J. Appl. Phys. 81 466-79

[48] Verhaart H, Tom J, Verhage A and Vos C 1987 Avalanches near solid insulators Proc. 5th Int. Symp. on HV Engineering (Braunschweig)

[49] Houle W A, Brown H M and Griffith O H 1979 Proc. Natl Acad. Sci. 76 4180-4
[50] Nijdam S, Wormeester G, van Veldhuizen E and Ebert U 2011 J. Phys. D: Appl. Phys. 44455201

[51] Pancheshnyi S, Eismann B, Hagelaar G and Pitchford L 2008 Computer code ZDPlasKin, University of Toulouse. LAPLACE Technical Report CNRS-UPS-INP, Toulouse, France (www.zdplaskin.laplace.univ-tlse.fr)

[52] Shcherbakov Y V and Sigmond R S 2007 J. Phys. D: Appl. Phys. 40 460-73

[53] Blom P P M, Smit C, Lemmens R H P and van Heesch E J M 1994 Gaseous Dielectr. VII 7 609-15

[54] Dilecce G, Ambrico P F and De Benedictis S 2010 J. Phys. D: Appl. Phys. $\mathbf{4 3} 195201$

[55] Shcherbakov Y V and Sigmond R S 2007 J. Phys. D: Appl. Phys. 40 474-87

[56] van Dijk J, Peerenboom K, Jimenez M, Mihailova D and van der Mullen J 2009 J. Phys. D: Appl. Phys. 42194012

[57] Gibalov V I and Pietsch G J 2000 J. Phys. D: Appl. Phys. 332618

[58] Gibalov V I and Pietsch G J 2012 Plasma Sources Sci. Technol. 21024010

[59] Kushner M J 2005 J. Phys. D: Appl. Phys. 381633

[60] Bhoj A N and Kushner M J 2004 J. Physics D: Applied Physics 372510

[61] Koren B 1993 A Robust Upwind Discretization Method for Advection, Diffusion and Source Terms (Amsterdam: Centrum voor Wiskunde en Informatica)

[62] Amestoy P R, Duff I S, L'Excellent J Y and Koster J 2001 SIAM J. Matrix Anal. Appl. 23 15-41

Amestoy P R, Guermouche A, L'Excellent J Y and Pralet S 2006 Parallel Comput. 32 136-56

[63] Vitello P, Penetrante B and Bardsley J 1994 Phys. Rev. E 495574 